\title{
Boundary Layer Instabilities Generated by Freestream Laser Perturbations
}

\author{
Amanda Chou* \\ NASA Langley Research Center, Hampton, VA, 23681, USA \\ Steven P. Schneider ${ }^{\dagger}$ \\ School of Aeronautics 83 Astronautics, Purdue University, West Lafayette, IN 47907, USA
}

\begin{abstract}
A controlled, laser-generated, freestream perturbation was created in the freestream of the Boeing/AFOSR Mach-6 Quiet Tunnel (BAM6QT). The freestream perturbation convected downstream in the Mach-6 wind tunnel to interact with a flared cone model. The geometry of the flared cone is a body of revolution bounded by a circular arc with a 3-meter radius. Fourteen PCB 132A31 pressure transducers were used to measure a wave packet generated in the cone boundary layer by the freestream perturbation. This wave packet grew large and became nonlinear before experiencing natural transition in quiet flow. Breakdown of this wave packet occurred when the amplitude of the pressure fluctuations was approximately $\mathbf{1 0 \%}$ of the surface pressure for a nominally sharp nosetip. The initial amplitude of the second mode instability on the blunt flared cone is estimated to be on the order of $10^{-6}$ times the freestream static pressure. The freestream laser-generated perturbation was positioned upstream of the model in three different configurations: on the centerline, offset from the centerline by $1.5 \mathrm{~mm}$, and offset from the centerline by $3.0 \mathrm{~mm}$. When the perturbation was offset from the centerline of a blunt flared cone, a larger wave packet was generated on the side toward which the perturbation was offset. The offset perturbation did not show as much of an effect on the wave packet on a sharp flared cone as it did on a blunt flared cone.
\end{abstract}

\section{Nomenclature}

\begin{tabular}{llll}
$f$ & frequency, $\mathrm{kHz}$ & \multicolumn{2}{l}{ Subscript } \\
$N$ & integrated growth factor & 0 & stagnation condition \\
$p$ & pressure, $\mathrm{kPa}$ & $i$ & initial condition \\
$r_{n}$ & nosetip radius, mm & $s$ & surface condition \\
$R e / m$ & freestream unit Reynolds number, $\mathrm{m}^{-1}$ & $\infty$ & freestream condition \\
$t$ & time after tunnel starts, $\mathrm{s}$ & \multicolumn{2}{l}{ Superscript } \\
$t_{p}$ & time after laser pulse is fired, $\mathrm{ms}$ & \multicolumn{1}{c}{ fluctuation } \\
$T$ & temperature, $\mathrm{K}$ & & \\
$x$ & distance from nosetip, $\mathrm{mm}$ & & \\
$\rho$ & density, $\mathrm{kg} / \mathrm{m}^{3}$ &
\end{tabular}

\section{Introduction}

Laminar-turbulent transition is not a well-understood process, yet it has a large impact on the design of hypersonic vehicles. Typically, this lack of knowledge is compensated for in the design process by overdesigning the hypersonic vehicle, which can make the mission cost-prohibitive. The alternative of accidentally under-designing the vehicle can lead to catastrophic failure. The design process can be optimized if transition can be better predicted.

\footnotetext{
*Research Aerospace Engineer, Flow Physics \& Control Branch, AIAA Senior Member.

${ }^{\dagger}$ Professor, AIAA Fellow.
} 
Current high-speed transition prediction methods are semi-empirical at best and incorporate only some physics. These methods estimate the transition process by examining the relative growth of instabilities. In a semi-empirical prediction method such as the $e^{N}$ method, ${ }^{1,2}$ the transition criterion is changed based on the freestream disturbance environment or model geometry and surface characteristics. Choosing transition criteria based only on the relative growth of instabilities may not take into consideration that the initial amplitude of the instability may be very large or very small. Furthermore, these semi-empirical methods generally only consider the linear growth of instabilities.

In 1975, Mack developed a forcing theory ${ }^{3}$ to apply to flat plate measurements taken at the Jet Propulsion Lab (JPL) in Pasadena, CA. ${ }^{4}$ This theory uses a forced response of the boundary layer to predict the growth of instabilities. The forcing theory was found to provide computations in good agreement with the measurements. However, the forced disturbances were distinctly different from the freestream disturbances. The mechanism by which the freestream disturbances became forced disturbances was not described with this theory, as Mack noted in Ref. 3. This disconnect in the theory remains an ongoing problem, and many measurements and computations need to be done to reconcile this gap.

The study of high-speed laminar-turbulent transition benefits from studies of receptivity, which describes how freestream disturbances enter the boundary layer to produce instabilities. Receptivity studies can be used to help determine the initial amplitudes of these boundary layer instabilities. If the receptivity process can be better understood, then an amplitude-based method of transition prediction can be better developed. Past computational and theoretical receptivity studies have included investigations of acoustic waves, ${ }^{5-7}$ of vorticity waves,${ }^{8}$ of discrete particles,${ }^{9}$ and of small entropic disturbances. ${ }^{10-14}$ However, receptivity experiments are difficult to perform, making it challenging to validate the computational and theoretical studies. Experimental studies have largely been in the subsonic regime, and the few existing high-speed studies typically use disturbances that are not easily characterized. Past high-speed experiments on the receptivity to freestream disturbances have included the use of an upstream electrode to generate acoustic disturbances ${ }^{15}$ and the use of a high-powered laser to generate a thermal disturbance. ${ }^{16-18}$

One of the most difficult elements in receptivity experiments lies in the ability to create well-defined, well-controlled disturbances. First, the disturbance must be repeatable and its amplitude and frequency content consistent. Second, the disturbance must not be so large that it causes bypass transition. Third, the equipment used to generate the upstream disturbance must not alter the overall freestream conditions (e.g., mean background noise level, Mach number, etc.). Lastly, in order to contribute to the development of amplitude-based prediction, the perturbation must be measurable so that the freestream disturbances can be characterized. The experimental approach used in this study was to create a controlled freestream perturbation in the Boeing/AFOSR Mach-6 Quiet Tunnel at Purdue University. Characteristics of this perturbation were then measured with fast pressure transducer probes and presented in Ref. 19. The perturbation was then allowed to convect downstream to a flared cone model, where its effect was measured with surface-mounted pressure transducers.

\section{The Facility and Equipment}

The Boeing/AFOSR Mach-6 Quiet Tunnel (BAM6QT) at Purdue University is a Ludwieg tube that can be run with laminar nozzle-wall boundary layers (Fig. 1). Conventional high-speed tunnels typically have turbulent nozzle-wall boundary layers that radiate acoustic noise into the freestream. These disturbances are not typically seen in flight and can cause transition to occur earlier in a ground test facility. Quiet facilities have a reduced freestream acoustic noise level more comparable to flight ${ }^{20-22}$ and provide a highly-controlled environment in which receptivity can be studied carefully. In the BAM6QT, the acoustic noise level has been measured at around $0.02 \%$ for about the first $2 \mathrm{~s}$ of run time. ${ }^{23,24}$ After $2 \mathrm{~s}$, the acoustic noise level increases slightly, and the run ends at about 4 or $5 \mathrm{~s}$. More information about the development of such quiet tunnels can be found in Ref. 22 .

To initiate a run in the BAM6QT, a set of diaphragms are burst downstream of the test section. This causes a shock wave to travel downstream into the vacuum tank and an expansion wave to travel upstream from the diaphragms. This expansion wave reflects between the end of the driver tube and the contraction about every $0.2 \mathrm{~s}$. This reflection causes a stair-step decrease in the stagnation pressure, stagnation temperature, and the unit Reynolds number. The decrease in stagnation pressure is less than $15 \%$ and the decrease in stagnation temperature is about $4 \%$ over the first $2 \mathrm{~s}$ of the run. A model installed in the tunnel starts at room temperature and remains at about the same temperature during these short runs. 


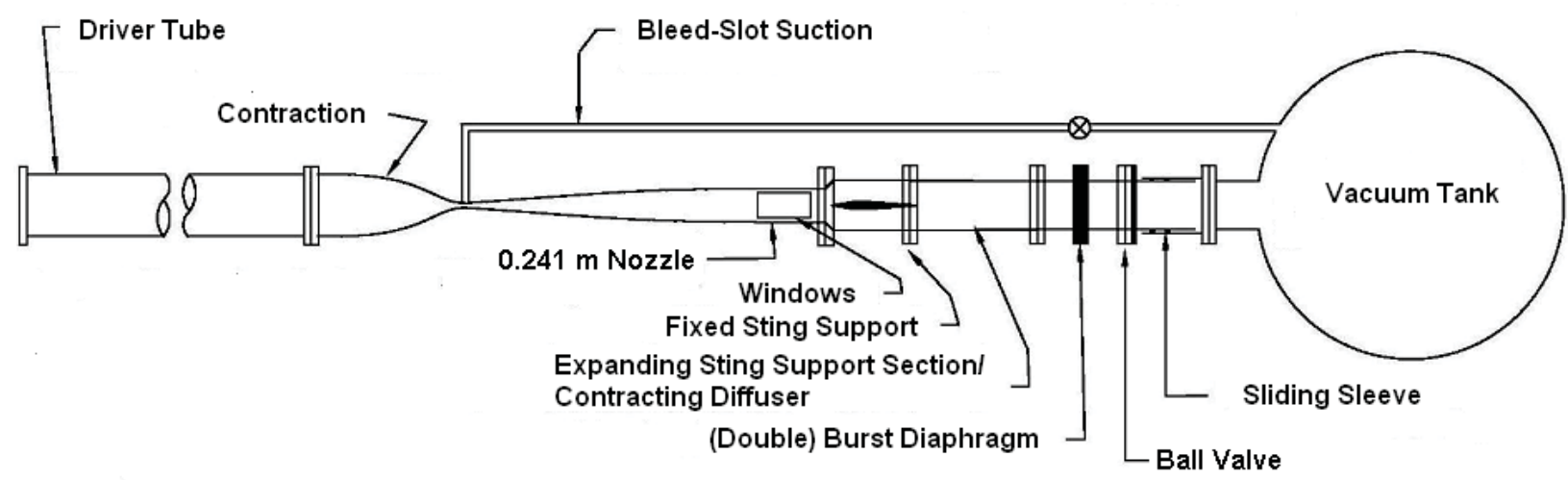

Figure 1. A schematic of the Boeing/AFOSR Mach-6 Quiet Tunnel.

\section{A. The Laser Perturber}

The laser perturber apparatus creates a perturbation by focusing a high-powered Nd:YAG laser to a small volume in the freestream of a wind tunnel. An ionized plasma is created at the focus of the optical system via laser-induced breakdown. A multiphoton absorption process drives the laser-induced breakdown in lowdensity environments such as a wind tunnel. ${ }^{25}$ The creation of the freestream perturbation through this process depends on the available molecules in the focal region as well as the photon flux. After ionization, the plasma cools and a weak shock emanates from the thermal core, but quickly decays. The thermal core is used as a controlled perturbation, which convects with the freestream and interacts with a test body downstream (Fig. 2).
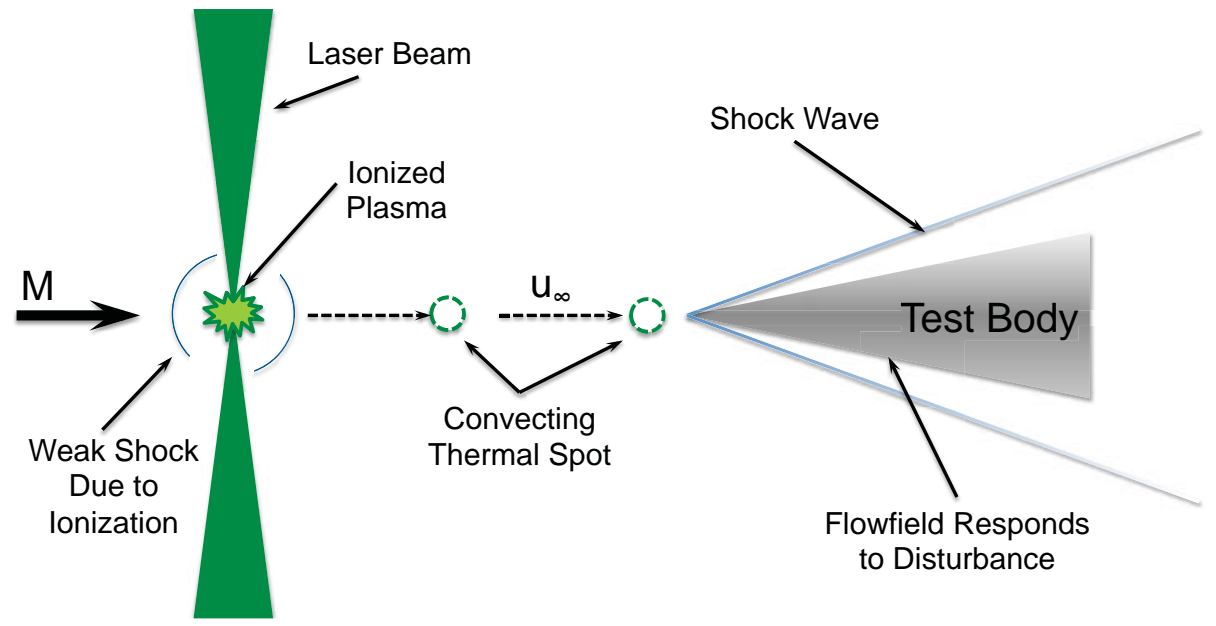

Figure 2. A schematic of the freestream perturbation relative to the test model.

The laser perturber apparatus consists of two main components: a Nd:YAG laser and a set of focusing optics. A Nd:YAG laser equipped with enhanced spatial mode and a laser seeder is used in this experimental setup. The beam diameter is about $4 \mathrm{~mm}$, with a pulse length of about $7 \mathrm{~ns}$. The maximum energy per pulse is typically around $270 \mathrm{~mJ}$. The laser pulses are fired at a rate of $10 \mathrm{~Hz}$, with each pulse lasting about $7 \mathrm{~ns}$. The laser is frequency-doubled so that it outputs light in the visible spectrum $(532 \mathrm{~nm})$ for laser eye safety.

A set of focusing optics was designed by Collicott ${ }^{26}$ to be used in the BAM6QT (Fig. 3). Rather than focusing the beam with a single lens, the beam diameter of the Nd:YAG laser was expanded prior to focusing. This expansion of the beam decreases the $f$-number $(f / D)$ of the lens system to create a tighter focus, which increases the photon flux in the focal region. The increase in photon flux aids in the breakdown process. This set of focusing optics consists of three air-spaced YAG triplets made by CVI/Melles-Griot: 
- a YAN-50.0-10.0 to expand the incoming laser beam

- a YAP-200.0-40.0 to collimate the beam

- and a YAP-200.0-40.0 to focus the beam inside the nozzle.

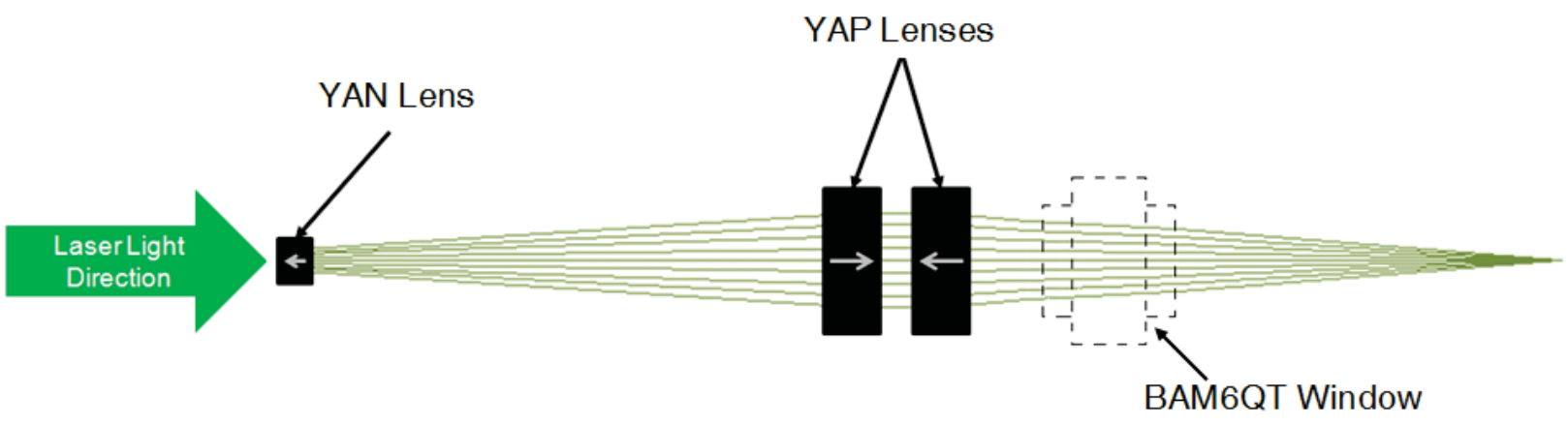

Figure 3. A schematic of the perturbation-forming optics used in the laser perturber apparatus. ${ }^{26}$

The freestream perturbation is expected to convect with the freestream velocity, which is nominally $874 \mathrm{~m} / \mathrm{s}$. Hot-wire measurements by Schmisseur ${ }^{16}$ showed that the perturbation in the Purdue Quiet-Flow Ludwieg Tube (a now-decommissioned Mach-4 quiet tunnel), was about $2 \mathrm{~mm}$ in diameter. Thus, the frequency response required to capture this perturbation may need to be over $1 \mathrm{MHz}$. Salyer ${ }^{27}$ modeled the perturbation as a spherical perturbation with a Gaussian distribution in density. He then used a laser differential interferometer (LDI) to characterize the perturbation in the same Mach-4 quiet tunnel. This measurement of the perturbation showed good agreement with the theoretical model.

Probe measurements of the perturbation were attempted at Mach-6 in the BAM6QT with a PCB 132A31 fast pressure transducer. ${ }^{19}$ These piezoelectric sensors have a 3.2 -mm diameter and a 0.76 -by-0.76-mm-square sensing element placed arbitrarily on the head of the sensor. These sensors provide only an AC-coupled measurement and are difficult to calibrate. A method of dynamic calibration of these sensors is currently being pursued by Berridge. ${ }^{28}$ Meanwhile, these measurements must use the factory-provided calibration.

Some spatial dimensions of the perturbation and the pitot pressure deficit across the perturbation were captured with the PCB 132A31 sensor (Fig. 4). Some jitter was present in the measurements due to variation in the freestream conditions from run to run. These measurements showed a large peak amplitude at the center of the perturbation of about a $65 \%$ deficit in pitot pressure. The diameter of the freestream disturbance was about $6 \mathrm{~mm}$. The disturbance also appeared to travel with the freestream velocity, so the disturbance takes about $9 \mu$ s to pass the probe. The peak magnitude of this perturbation may be considered large, but the integrated effect of the discrete perturbation may be relatively small due to its short duration. Further details on the characterization measurements of this disturbance can be found in Refs. 16, 27, 19, and 29.

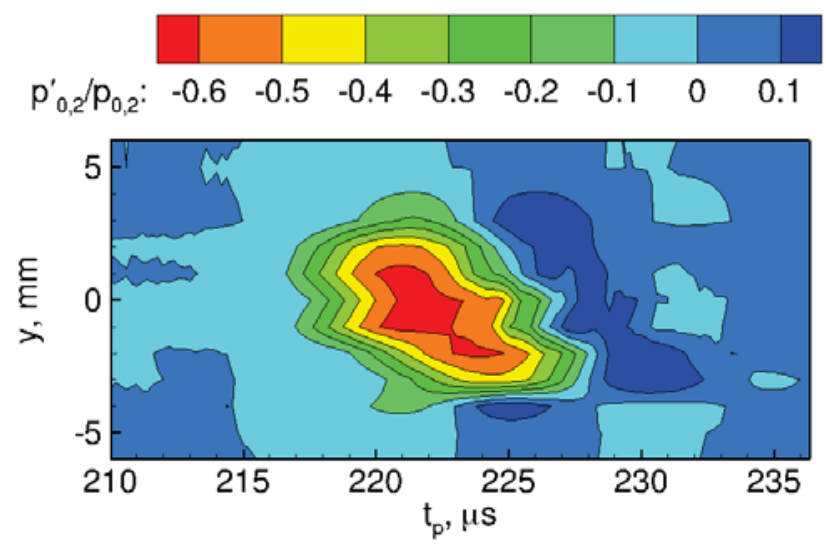

Figure 4. Measurements of a laser-generated perturbation in the freestream of the BAM6QT with a PCB probe. Probe is placed $\mathbf{2 1 9 . 0} \mathbf{m m}$ upstream of where the perturbation is generated. $p_{0}=1022 \mathrm{kPa}, T_{0}=412.8 \mathrm{~K}$, $\rho_{\infty}=0.040 \mathrm{~kg} / \mathrm{m}^{3}$. 


\section{B. The Flared Cone Model}

The model used for this experiment was a flared cone (Fig. 5). The geometry of the flared cone is a body of revolution bounded by a circular arc with a 3 -m radius. The cone frustum was manufactured on a CNC lathe out of 6061-T6 aluminum round stock. The nosetips for the model are interchangeable and manufactured out of 17-4PH stainless steel round stock. Two nosetips are available for this model: a 1-mm-radius (blunt) nosetip (Fig. 6(a)) and a 0.16-mm-radius (sharp) nosetip (Fig. 6(b)). The nose tips are designed to be hemispherical and the curvature of the nosetip lies tangent to the circular-arc flare. The different nosetips have different lengths, so the position of the installed sensors relative to the nose tip changes.

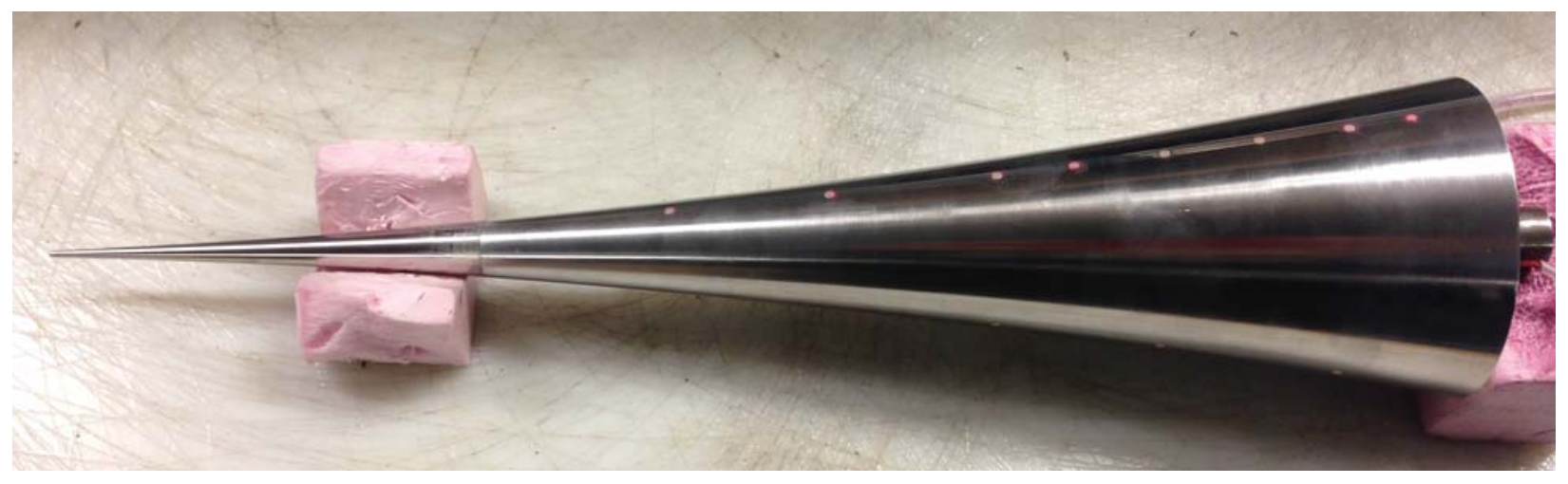

Figure 5. A photograph of the 3-m-circular-arc flared cone and PCB sensors.

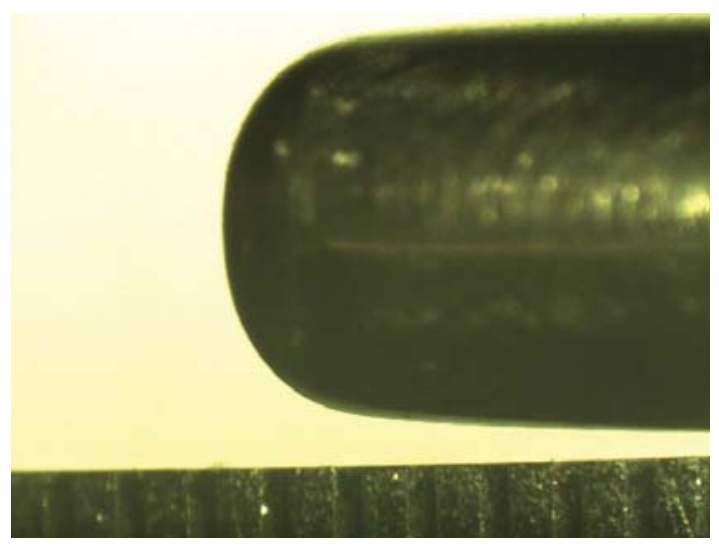

(a) Blunt nose: $r_{n}=1 \mathrm{~mm}$.

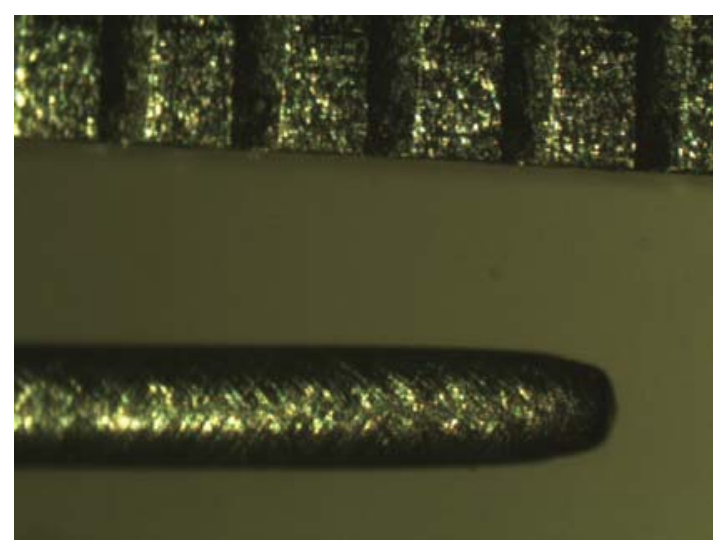

(b) Sharp nose: $r_{n}=0.16 \mathrm{~mm}$.

Figure 6. Close-up of nosetips used for experiment. Ruler divisions shown in the image have a 1/64-in. (0.40-mm) spacing.

A total of fourteen PCB 132A31 fast pressure transducers were installed in the cone. Eight of these fast pressure transducers were installed along the $0^{\circ}$ ray, which faced $180^{\circ}$ away from the incoming $\mathrm{Nd:YAG}$ laser beam. Three sensors were installed on the $+120^{\circ}$ and $-120^{\circ}$ rays each to provide three axial rays and three stations of azimuthal sensor arrays. The azimuthal arrays of sensors were used both to align the cone model and to study the effects of an offset laser-generated perturbation. The data were recorded with three digital phosphor oscilloscopes, which can each record up to four different data channels. The maximum recording length of each of these channels is up to 250 million points. Two of the oscilloscopes have an analog bandwidth of $500 \mathrm{MHz}$ and one of the oscilloscopes has an analog bandwidth of $1 \mathrm{GHz}$. The sensors were sampled at $2 \mathrm{MHz}$ for the entire run. The data were acquired with the "High-Res Mode" feature on the oscilloscope, which samples the data at the maximum bandwidth and then filters and records the data at the user-specified sampling frequency. Due to the limitation in the number of long-memory oscilloscopes, only a total of 12 sensors could be monitored in any given run.

The flared cone was originally designed by Wheaton and Juliano in 2009 to generate large second-mode 
waves. ${ }^{30}$ Sample power spectra for the blunt and sharp flared cone are shown in Fig. 7 at a unit Reynolds number of about $10 \times 10^{6} / \mathrm{m}$. No freestream laser perturbations were generated in the BAM6QT for these data. Figure 7(a) shows that the second mode is large and a possible harmonic appears in the spectra at $x=200$ and $302 \mathrm{~mm}$. Breakdown starts to occur before $x=403 \mathrm{~mm}$, because there is an increase in the broadband frequency content. Later, extra sensor ports were added to the model, to better monitor the growth of the boundary layer instabilities along a single ray. Figure $7(\mathrm{~b})$ shows the power spectra of the PCB measurements on the sharp nosetip after some of these extra sensor ports were added. The second mode grows on the sharp model between $x=231 \mathrm{~mm}$ and $332 \mathrm{~mm}$. The natural waves are nonlinear at $x=382 \mathrm{~mm}$, as evidenced by the presence of large harmonics. These large harmonics also coincide with the presence of streaks of higher heating, as shown in Fig. 8. These streaks are suspected to be related to a nonlinear breakdown mechanism. ${ }^{31}$ Details on previous measurements without the freestream laser perturbation for the blunt nosetip model are available in Ref. 30 and similar measurements with the sharp nosetip model are available in Refs. 32 and 33.

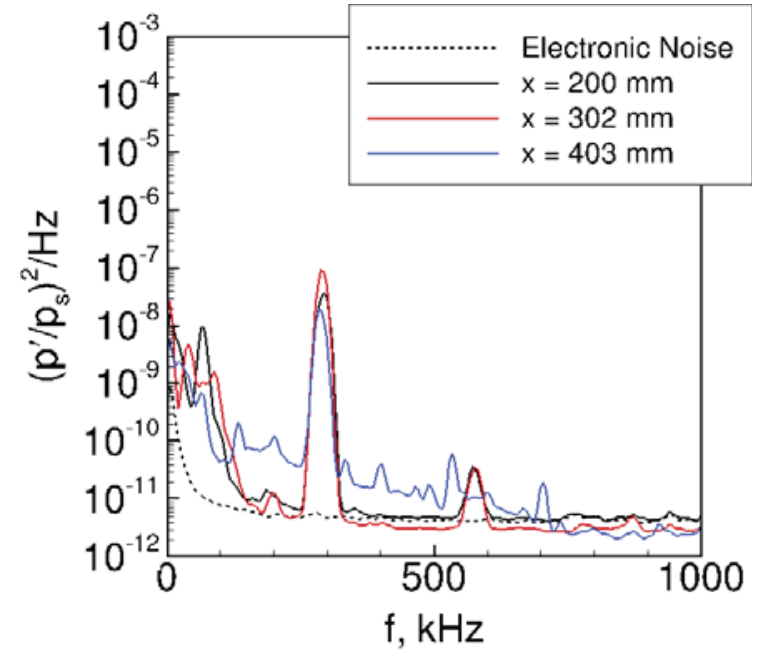

(a) Blunt nose: $r_{n}=1 \mathrm{~mm}, p_{0}=946.1 \mathrm{kPa}, T_{0}=426.6 \mathrm{~K}$, $\operatorname{Re} / \mathrm{m}=10.3 \times 10^{6} / \mathrm{m}^{30}$

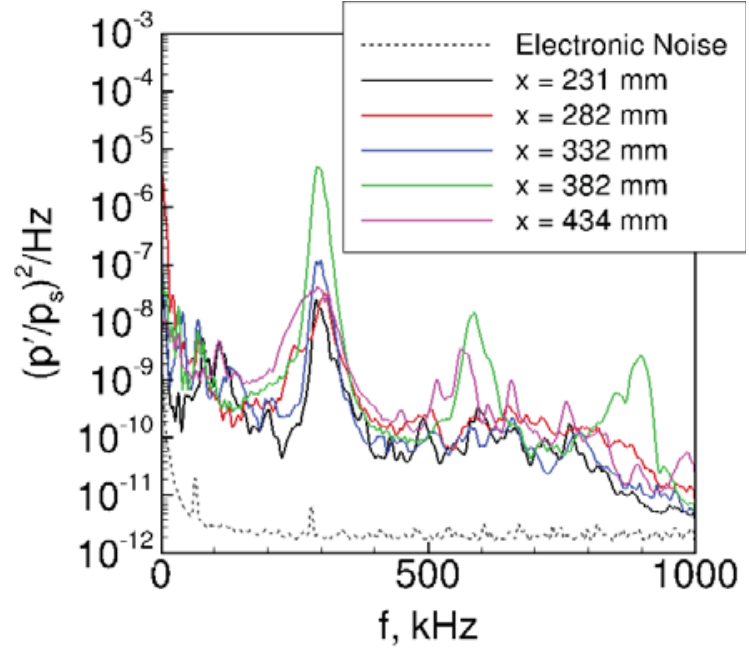

(b) Sharp nose: $r_{n}=0.16 \mathrm{~mm}, p_{0}=962.1 \mathrm{kPa}, T_{0}=$ $423.4 \mathrm{~K}, \mathrm{Re} / \mathrm{m}=10.7 \times 10^{6} / \mathrm{m}^{32}$

Figure 7. Sample power spectra of PCB measurements on the flared cone. No laser perturbations are present in the freestream.

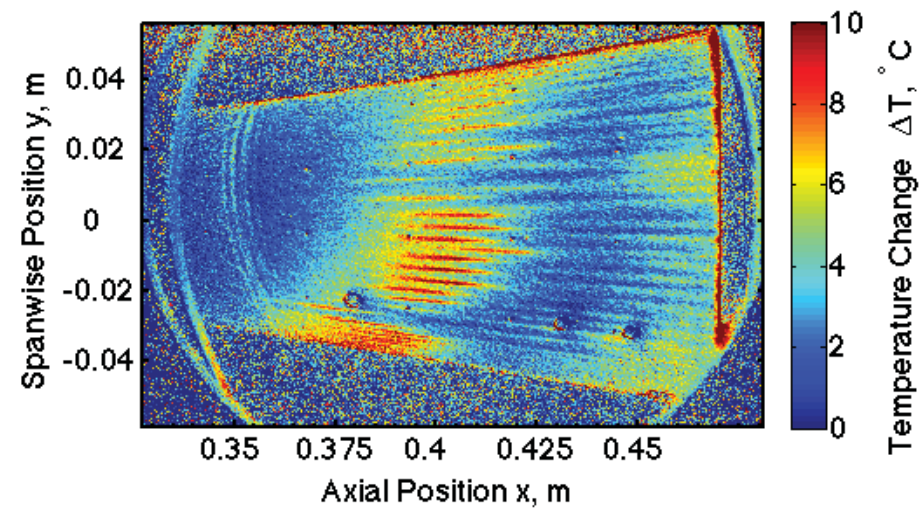

Figure 8. Temperature-sensitive paints measurements corresponding to data in Figure $7(\mathbf{b}) \cdot r_{n}=0.16 \mathrm{~mm}$, $p_{0}=962.1 \mathrm{kPa}, T_{0}=423.4 \mathrm{~K}, R e / m=10.7 \times 10^{6} / \mathbf{m} .^{33}$ 


\section{Results}

Run conditions for this model were limited to a small range of Reynolds numbers due to several factors. Initial stagnation pressures lower than about $500 \mathrm{kPa}$ caused boundary-layer separation on the nozzle wall of the BAM6QT. Wave packets generated by the laser perturbation broke down at the aft end of the model for stagnation pressures of about $670 \mathrm{kPa}$ on the sharp model and about $820 \mathrm{kPa}$ on the blunt model. For the blunt nosetip model, pressures lower than about $670 \mathrm{kPa}$ produced small wave packets that were unable to be measured by most of the sensors. Nevertheless, a large amount of data were acquired in this experiment.

The conditions at which both models could be used had little overlap. The tunnel stagnation temperature and pressure decrease quasi-statically as the run time increases. The times at which both models had overlapping stagnation or freestream conditions were at the end of the run on the blunt model and near the start of the run on the sharp model. However, these two time periods may not be suitable for data analysis. At the beginning of a run, the flow may not be fully established. At the end of a run, the number of turbulent bursts increases and an unexplained increase in noise occurs. ${ }^{24}$ These circumstances would not be representative of the flow during a majority of the run.

It is assumed that the freestream density drives the formation of the laser perturbation. ${ }^{19}$ Thus, the freestream density was matched to compare the effect of the laser perturbation on the two different nosetips. As a result, the stagnation pressures may be as much as $10 \%$ different between the two different nosetip cases. The stagnation temperature and Reynolds numbers will differ by as much as $6 \%$.

\section{A. Axial Development of a Wave Packet in the Boundary Layer}

The axial development of the wave packet can be monitored using sensors installed along the $0^{\circ}$ ray. The freestream laser perturbation was aligned to the centerline of the flared cone model for these measurements. This alignment setup was expected to produce an axisymmetric disturbance (wave packet) in the cone boundary layer.

Figure 9 shows some examples of the evolution of a single wave packet in the streamwise direction for both nosetips at $\rho_{\infty}=0.026 \mathrm{~kg} / \mathrm{m}^{3}$. These plots show the effect of a single laser-generated perturbation (one laser shot) on a blunt flared cone (Fig. 9(a)) and on a sharp flared cone (Fig. 9(b)). The time traces from a surface pressure transducer are offset by an amount proportional to the axial distance from the cone nosetip to the center of the sensor.

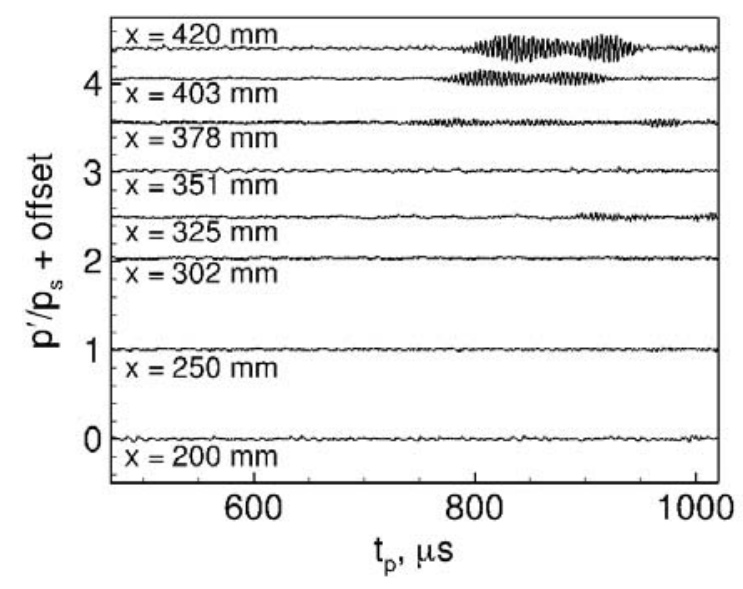

(a) $r_{n}=1 \mathrm{~mm} \cdot p_{0}=599.5 \mathrm{kPa}, T_{0}=411.1 \mathrm{~K}, R e / m=$ $6.98 \times 10^{6} / \mathrm{m}$.

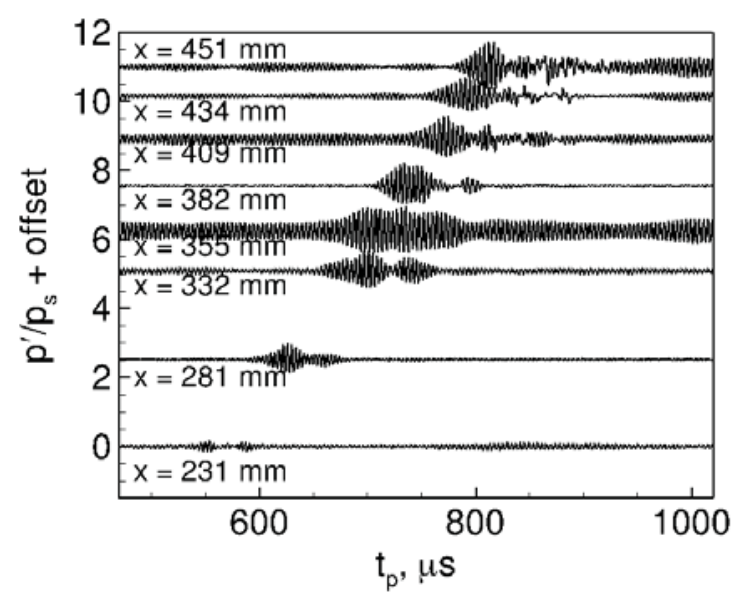

(b) $r_{n}=0.16 \mathrm{~mm} . p_{0}=534.3 \mathrm{kPa}, T_{0}=428.3 \mathrm{~K}, R e / m=$ $6.59 \times 10^{6} / \mathrm{m}$.

Figure 9. Axial development of a laser-generated wave packet in the boundary layer of a flared cone for $\rho_{\infty}=0.026 \mathrm{~kg} / \mathrm{m}^{3}$.

On the blunt flared cone (Fig. 9(a)), a wave packet is not detectable in the surface pressure measurements until about $x=378 \mathrm{~mm}$. The wave packet grows larger with increasing distance from the nosetip, but still remains fairly small. The envelope of the disturbance also appears to consist of multiple bursts, with the first double-burst feature appearing at $x=378 \mathrm{~mm}$. Transition is not observed at this freestream density for the 
blunt nosetip. On the sharp flared cone (Fig. 9(b)), the wave packet first appears at $x=231 \mathrm{~mm}$ and grows large until about $x=355 \mathrm{~mm}$. Note that the scaling of the vertical axis in Fig. 9(b) is almost 3 times greater than the vertical axis in Fig. 9(a) due to the increase in wave packet amplitudes. A distinct double-burst feature is present on the sharp model between $x=231 \mathrm{~mm}$ and $382 \mathrm{~mm}$. After $x=355 \mathrm{~mm}$, the wave packet amplitude appears to decay, and begins to show characteristics of breakdown at about $x=434 \mathrm{~mm}$.

These time traces show a distinct difference in the effect of nosetip bluntness. The amplitude of the wave packet on the blunt nosetip is very small and the wave packet does not break down at the aft end of the model. The sharp nosetip shows the effect of a freestream laser-generated perturbation at even the first sensor location and breakdown begins to occur near the aft end of the cone. The wave packet amplitude is much larger on the sharp cone, indicating either faster growth of the instability or increased receptivity. This effect of nosetip bluntness was seen previously by Balakumar in his computational studies. ${ }^{34}$ However, those computations were performed with acoustic plane waves rather than a discrete thermal disturbance.

\section{B. Frequency Content of Wave Packets on the Flared Cone}

The spectral content of these packets can be determined by taking a FFT of the time trace. FFTs of the response to multiple laser perturbations must be averaged to obtain an accurate estimate of the power spectra. The number of usable laser shots is limited by the presence of turbulent spots on the nozzle wall, nozzle wall separation, and the duration of the tunnel run. The freestream Reynolds number also decreases as the run time increases due to the reflections of the expansion wave. Thus, the conditions at which each laser shot occurs might not be the same.

To illustrate the effect that the changing conditions have on the power spectra, a FFT was applied to four different laser shots at different times during a single tunnel run. These FFTs are shown in Figure 10. A 1000-point Blackman window was applied to each wave packet and the spectral content of this window was estimated using Welch's method. The FFTs are all fairly similar, with small changes in the second-mode amplitude. The largest difference in the FFTs occurs between the first shot in a run and the last usable shot in a run. Between these two shots, there is at most a $30 \%$ difference in the peak power and a $16 \%$ difference in the RMS amplitude. While this is not ideal, averaging occurs across consecutive laser shots and qualitative comparisons can still be made from the averaged spectra.

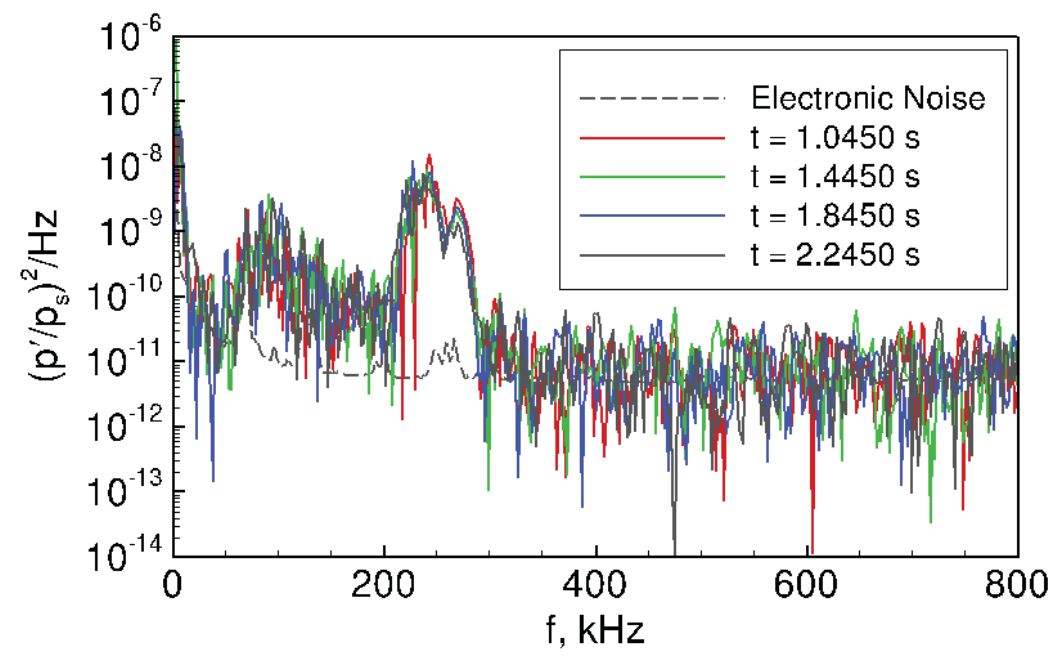

Figure 10. Example FFTs of different laser shots taken at different times during a tunnel run. $r_{n}=0.16 \mathrm{~mm}$, $p_{0}=534.3 \mathrm{kPa}, T_{0}=428.3 \mathbf{K}, R e / m=5.81 \times 10^{6} / \mathbf{m}, x=231 \mathbf{m m}$.

A frequency analysis of the data displayed in Fig. 9 is shown in Fig. 11. These spectra use three FFTs each because the intent was to compare only measurements at the same freestream density. This overlap in freestream density only occurred across three laser shots. As shown previously in Fig. 10, the characteristics of the FFTs are fairly similar from shot to shot. The spectra were smoothed with a zero-phase averaging filter to provide clearer data.

Figure 11(a) shows the power spectra for the blunt flared cone. The spectra show a large fundamental peak at the expected second-mode frequency at about $240 \mathrm{kHz}$. A higher harmonic at about $480 \mathrm{kHz}$ may 


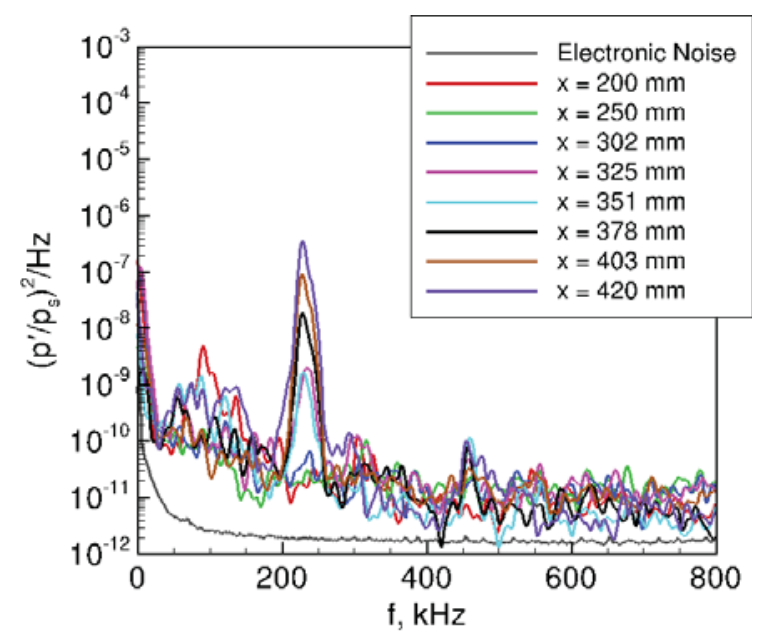

(a) $r_{n}=1 \mathrm{~mm} \cdot p_{0}=599.5 \mathrm{kPa}, T_{0}=411.1 \mathrm{~K}, R e / m=$ $6.98 \times 10^{6} / \mathrm{m}$.

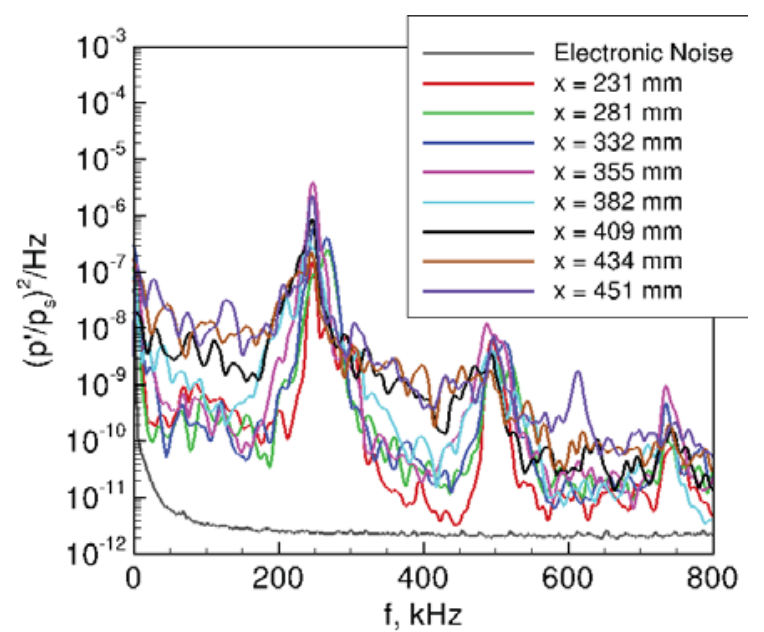

(b) $r_{n}=0.16 \mathrm{~mm} . p_{0}=534.3 \mathrm{kPa}, T_{0}=428.3 \mathrm{~K}, R e / m=$ $6.59 \times 10^{6} / \mathrm{m}$.

Figure 11. Frequency content of the wave packets initiated by a freestream laser-generated perturbation at $\rho_{\infty}=0.026 \mathrm{~kg} / \mathrm{m}^{3}$.

also be present for sensor stations aft of $x=351 \mathrm{~mm}$. The wave packet was not detectable in the time traces until about $x=378 \mathrm{~mm}$, which is aft of the station where a harmonic first appears in the power spectra. Currently, it is unclear if the second mode peaks in the spectra are related to the large natural waves present on the cone, to the presence of the wave packet, or to both.

Figure 11(b) shows the spectral content of the wave packet on the sharp flared cone. At the most-upstream sensor $(x=231 \mathrm{~mm})$, the spectral content is characterized by a large fundamental peak at about $247 \mathrm{kHz}$, a harmonic at $493 \mathrm{kHz}$, and a higher harmonic at $740 \mathrm{kHz}$. The presence of these harmonics indicates that the wave packet amplitude is already large and may be nonlinearly saturated. The spectral content at $x=382 \mathrm{~mm}$ indicates the possible onset of transition because the second-mode peak has begun to increase in bandwidth and decrease in amplitude. Breakdown occurs around $x=409 \mathrm{~mm}$, as indicated by an increased level in the broadband frequencies.

\section{RMS Amplitudes}

The RMS amplitude of the second-mode wave was calculated by integrating the spectra in a 20-kHz frequency band centered around the second-mode peak frequency, then taking the square root. These RMS amplitudes are then compared to the linear $N$-factor computations of the second mode performed previously. ${ }^{30,32}$ The flare of the cone creates a pressure gradient, so the pressure along the surface of the cone changes slightly with each axial station. Thus, the RMS amplitudes are not normalized by the computed surface pressure in this section, except where indicated.

Instead, the absolute RMS amplitudes are normalized by the absolute RMS amplitude of the measured second mode at the first sensor station. This normalization is done to better compare the RMS amplitudes to the computed $N$-factor. The $N$-factor is defined as:

$$
N=\ln \frac{A}{A_{0}}
$$

where $A$ is the amplitude of the instability at a given location and $A_{0}$ is the initial instability amplitude. The RMS amplitudes are normalized by the calculated RMS amplitude at the first sensor location because the initial amplitude is unknown. The relationship between the normalized RMS amplitude $\left(A / A_{1}\right)$ and the $N$-factor is given by

$$
N=\ln \left(\frac{A}{A_{1}} \frac{A_{1}}{A_{0}}\right)=\ln \frac{A}{A_{1}}+\ln \frac{A_{1}}{A_{0}}
$$

where $A_{1}$ is the amplitude at the first sensor station. The natural logarithm of the normalized RMS amplitude will be the same scale as the $N$-factor with some offset defined by the $N$-factor at the first sensor station. 
The computations were for a higher stagnation pressure than any of the experimental conditions. Computations for each nosetip were completed prior to the experiment: in 2009 for the blunt nosetip ${ }^{30}$ and in 2010 for the sharp nosetip. ${ }^{32}$ The conditions used in the computations could not be matched experimentally due to limitations in the useful range of run conditions. However, measurements with the closest conditions to the computations are used for a qualitative comparison between the experiments and computations. For the blunt nosetip case, the computations correspond to a different set of measurements than those shown previously. The measurements which correspond most closely to the computations are shown in Fig. 12. The unit Reynolds number for these measurements is higher, and thus, the wave packet disturbance is larger. Figure 12(a) shows that the wave packet has a multi-burst structure between $x=378 \mathrm{~mm}$ and $420 \mathrm{~mm}$. The spectra in Fig. 12(b) also show that the second mode does not break down at the most aft sensor station of $x=420 \mathrm{~mm}$. The background frequency content remains at about the same level on all of the sensors.

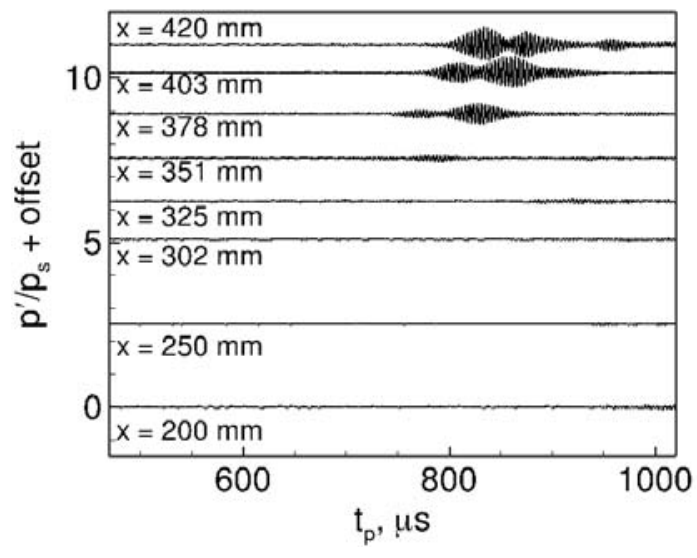

(a) Time series.

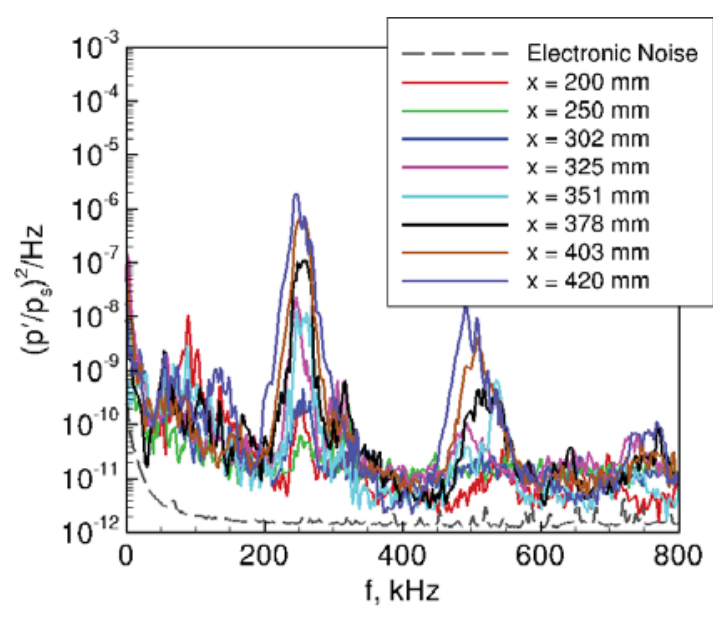

(b) Power spectral density.

Figure 12. Measurements of a wave packet generated on a blunt flared cone. $p_{0}=740.3 \mathbf{k P a}, T_{0}=428.0 \mathrm{~K}$, $R e / m=7.86 \times 10^{6} / \mathbf{m}, \rho_{\infty}=0.030 \mathrm{~kg} / \mathbf{m}^{3}$.

Figure 13 shows a comparison of the measurements from the surface-mounted pressure transducers to the $N$-factor computed by STABL from Refs. 30 and 32. The computed $N$-factor curves are given by solid lines, which correspond to the left-hand axes in Fig. 13. The natural logarithm of the normalized RMS values are given by open squares and correspond with the right-hand axes in Fig. 13. The RMS amplitudes normalized by the surface pressure are given in Tables 1 and 2 with a comparison to the $N$-factors from the STABL computations in Refs. 30 and 32.

Table 1. RMS amplitudes measured with PCB sensors and N factors from STABL computations for blunt flared cone.

\begin{tabular}{cccc}
\hline $\begin{array}{c}\text { Distance from } \\
\text { Nosetip } \\
x, \mathbf{m m}\end{array}$ & $\begin{array}{c}\text { Normalized } \\
\text { RMS Amplitude } \\
\left(p^{\prime} / p_{s}\right) \times 10^{2}\end{array}$ & $\begin{array}{c}\text { Natural Logarithm of } \\
\text { Normalized RMS Amplitude } \\
\ln \left(p^{\prime} / p_{x=200 \mathrm{~mm}}^{\prime}\right)\end{array}$ & $\begin{array}{c}\text { Computed } \\
N \text { Factor } \\
(\text { Reference 30) }\end{array}$ \\
\hline \hline 200 & 0.212 & 0 & 2.4 \\
250 & 0.186 & -0.0145 & 4.8 \\
302 & 0.392 & 0.851 & 7.6 \\
325 & 0.954 & 1.79 & 9.0 \\
351 & 2.67 & 2.89 & 10.6 \\
378 & 9.38 & 4.20 & 12.2 \\
403 & 14.0 & 4.66 & 13.8 \\
420 & 10.1 & 4.37 & 14.8 \\
\hline
\end{tabular}

The right-hand axis of Figure 13(a) is shifted so that the measured RMS amplitude at the third sensor location $(x=302 \mathrm{~mm})$ falls on the same curve as the computation. The first three sensor locations are 


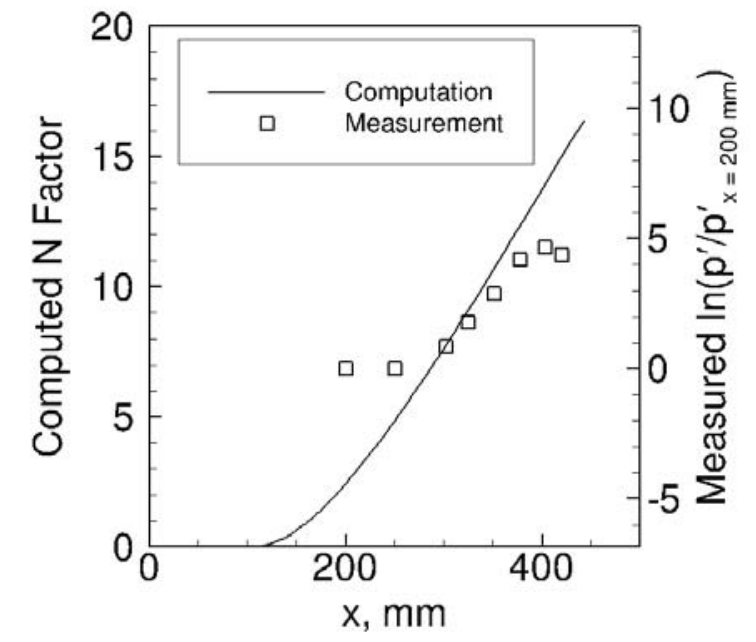

(a) $r_{n}=1 \mathrm{~mm}$. Computation: $p_{0}=965.3 \mathrm{kPa}, T_{0}=433 \mathrm{~K}$, $\rho_{\infty}=0.040 \mathrm{~kg} / \mathrm{m}^{3}$. Experiment: $p_{0}=740.3 \mathrm{kPa}, T_{0}=$ $428.0 \mathrm{~K}, R e / m=7.86 \times 10^{6} / \mathrm{m}, \rho_{\infty}=0.030 \mathrm{~kg} / \mathrm{m}^{3}$.

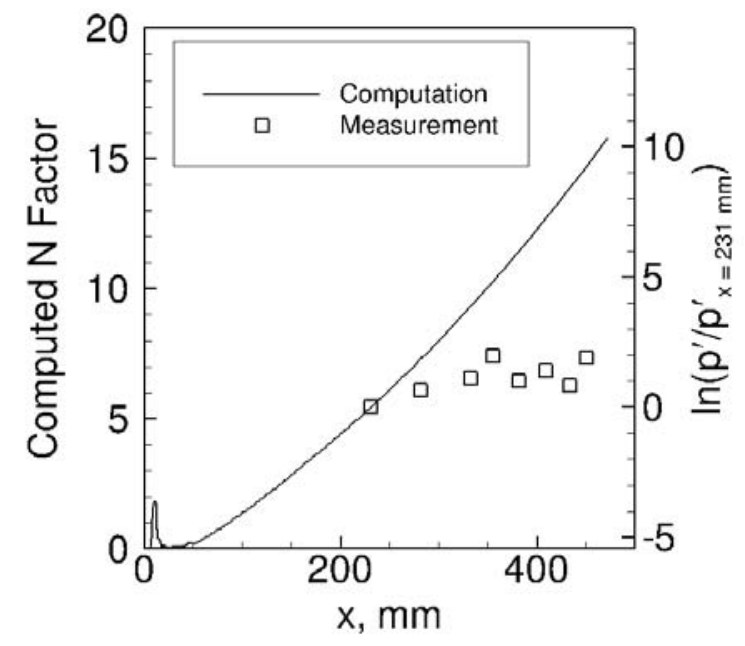

(b) $r_{n}=0.16 \mathrm{~mm}$. Computation: $p_{0}=620.5 \mathrm{kPa}, T_{0}=$ $433 \mathrm{~K}, \rho_{\infty}=0.026 \mathrm{~kg} / \mathrm{m}^{3}$. Experiment: $p_{0}=534.3 \mathrm{kPa}$, $T_{0}=428.3 \mathrm{~K}, \operatorname{Re} / m=6.59 \times 10^{6} / \mathrm{m}, \rho_{\infty}=0.022 \mathrm{~kg} / \mathrm{m}^{3}$.

Figure 13. A comparison of the measured RMS amplitude of the second-mode wave (right-hand axis) with the computed $N$ factor (left-hand axis).

Table 2. RMS amplitudes measured with PCB sensors and N factors from STABL computations for blunt flared cone.

\begin{tabular}{cccc}
\hline $\begin{array}{c}\text { Distance from } \\
\text { Nosetip } \\
x, \mathbf{m m}\end{array}$ & $\begin{array}{c}\text { Normalized } \\
\text { RMS Amplitude } \\
\left(p^{\prime} / p_{s}\right) \times 10^{2}\end{array}$ & $\begin{array}{c}\text { Natural Logarithm of } \\
\text { Normalized RMS Amplitude } \\
\ln \left(p^{\prime} / p_{x=231}^{\prime} \mathrm{mm}\right)\end{array}$ & $\begin{array}{c}\text { Computed } \\
N \text { Factor } \\
\text { (Reference 32) }\end{array}$ \\
\hline \hline 231 & 3.75 & 0 & 5.4 \\
281 & 6.30 & 0.632 & 7.3 \\
332 & 8.93 & 1.10 & 9.3 \\
355 & 20.4 & 1.98 & 10.2 \\
382 & 7.33 & 1.01 & 11.4 \\
409 & 10.1 & 1.40 & 12.6 \\
434 & 5.30 & 0.811 & 13.8 \\
451 & 14.9 & 1.88 & 14.6 \\
\hline
\end{tabular}

suspected to measure only the electronic noise floor because the measured amplitudes at these locations are small and similar. Only three or four points seem to agree with the computation, leaving the last few sensor locations to show growth to be less than predicted by the linear computation. The portion of the measured data that falls near the same curve as the computation probably corresponds to a small region of linear growth. The leveling out of RMS amplitudes at the aft end of the cone is probably due to nonlinear saturation of the wave packet. Break down of the wave packet is not observed in the blunt flared cone data in Figure 12(b).

The right-hand axis of Figure 13(b) is shifted so that the measured RMS amplitude at the first sensor location falls on the computational curve. All the amplitudes of the second-mode wave packet in this case are relatively similar. This is likely due to nonlinear saturation, which was indicated by the large harmonic amplitudes shown in the power spectra in Fig. 11(b). It is possible that the installed sensors do not capture the linear growth region because the power spectra at the first sensor location $(x=231 \mathrm{~mm})$ shows the presence of harmonics of the second-mode instability. The power spectra in Figure 11(b) show that the packet begins to break down near $x=382$ or $409 \mathrm{~mm}$, where the amplitude of the second mode is about $7-10 \%$ of the mean surface pressure. 
A comparison of the measurements and the computations is only qualitative. However, a rough estimate of the initial amplitudes of the second-mode instability can be obtained by comparing a point in the linear growth region of the measurements to the computations. On the blunt flared cone, this point was located at $x=302 \mathrm{~mm}$. The inferred initial amplitude of the second mode on the blunt flared cone is $p_{i} / p_{\infty}=$ $3.51 \times 10^{-6}$, where $p_{i}$ is the initial amplitude and $p_{\infty}$ is the freestream static pressure. This amplitude is very small compared to the large peak amplitude of the freestream laser perturbation, which provided more than a $65 \%$ peak deficit in the pitot pressure. On the sharp flared cone, it is unclear that there is any linear growth region. Thus, such a comparison to the linear computation will not be performed here.

Previous computational studies using planar acoustic waves of similar magnitude to the freestream laser perturbation suggested that bypass transition would occur. Other computational studies have shown that small discrete thermal disturbances can create wave packets of similar amplitude to what was observed in this experiment. ${ }^{14}$ Computations have not yet used a large, discrete thermal perturbation, but the previous studies suggest that such a perturbation might cause bypass transition. The experiments show that this is not the case and small instabilities can be generated by large, discrete disturbances in the freestream.

\section{Azimuthal Measurements of a Wave Packet in the Boundary Layer}

Azimuthal measurements of the wave packet were taken at three different axial stations along the cone frustum. These three axial stations each had three sensors installed $120^{\circ}$ apart. The azimuthal variation of the wave packet was studied by aligning the perturbation relative to the cone centerline in three different configurations. The perturbation was first aligned to the centerline and later offset by $1.5 \mathrm{~mm}$ and by $3.0 \mathrm{~mm}$ from the centerline. The perturbation alignment offset was toward each ray of sensors, at azimuthal positions of $0^{\circ},+120^{\circ}$, and $-120^{\circ}$. The time traces corresponding to each sensor ray are offset by an amount proportional to the azimuthal position. The power spectral densities shown in this section are estimated using Welch's method to determine FFTs of each wave packet and 15 different FFTs are averaged together.

Figures 14 and 15 show the time response and the power spectral densities of the measurements made on the blunt flared cone, respectively. The freestream perturbation is aligned to the cone centerline for the measurements shown. Figure 14(a) shows that the wave packets are not discernible from the natural secondmode waves at the first sensor station. The amplitude of the waves increases slightly around $850 \mu \mathrm{s}$ after the laser pulse is fired. The cause of this increase is unknown, because it appears at every sensor station at the same time. The power spectra of the measurements at the most upstream azimuthal array $(x=302 \mathrm{~mm})$ show a peak from the second-mode instability in Fig. 15(a) at about $250 \mathrm{kHz}$. The wave packet becomes more apparent in the time traces starting at the second azimuthal array located at $x=351 \mathrm{~mm}$ (Fig. 14(b)). The power spectra in Fig. 15(b) show that the first harmonic of the second mode is now present in all of the time traces at this axial station. Figure 14(c) shows that the wave packets grow larger as they travel downstream, and are all about the same shape. The amplitudes of the wave packets are slightly different, which may result from a very slight misalignment of the freestream disturbance relative to the cone centerline. The spectra in Fig. 15(c) show that the amplitudes and frequency bands for the second mode appear to be fairly similar around the azimuth at $x=403 \mathrm{~mm}$.

Figures 16 and 17 show the time response and power spectral densities of the measurements made on the sharp flared cone, respectively. Again, the freestream perturbation is aligned to the cone centerline for these measurements. The wave packet is apparent in the time traces on the sharp cone at all three axial stations and each azimuthal station (Fig. 16). The vertical scaling of these plots is 2.5 times the vertical scaling of the plots in Fig. 14 because the wave packets are much larger on the sharp flared cone than on the blunt flared cone. The first harmonic of the second mode is present in the power spectra of the measurements at the most upstream azimuthal array at $x=332 \mathrm{~mm}$ (Fig. 17(a)). Higher harmonics appear in the spectra at the next azimuthal array at $x=382 \mathrm{~mm}$ (Fig. 17(b)). The presence of these large harmonics in each of the azimuthal arrays indicates that the wave packet may already be nonlinearly saturated at each of these stations. Thus, small changes to the amplitude of the wave packet may not be as evident as they were on the blunt cone. The harmonics begin to disappear from the spectra at the most downstream of the azimuthal arrays at $x=434 \mathrm{~mm}$ (Fig. 17(c)). The background frequency content also begins to rise, indicating that the wave packet begins to break down near $x=434 \mathrm{~mm}$.

The freestream perturbation was next placed $1.5 \mathrm{~mm}$ off of the centerline upstream of the model to determine the effect of a purposefully misaligned perturbation. Figures 18 and 19 show the effect of the offset perturbation on the blunt flared cone. The perturbation is offset toward the $0^{\circ}$ ray of sensors. As seen in Fig. 18(a), the wave packet is not easily discernible from the natural second-mode waves at $x=302 \mathrm{~mm}$ 


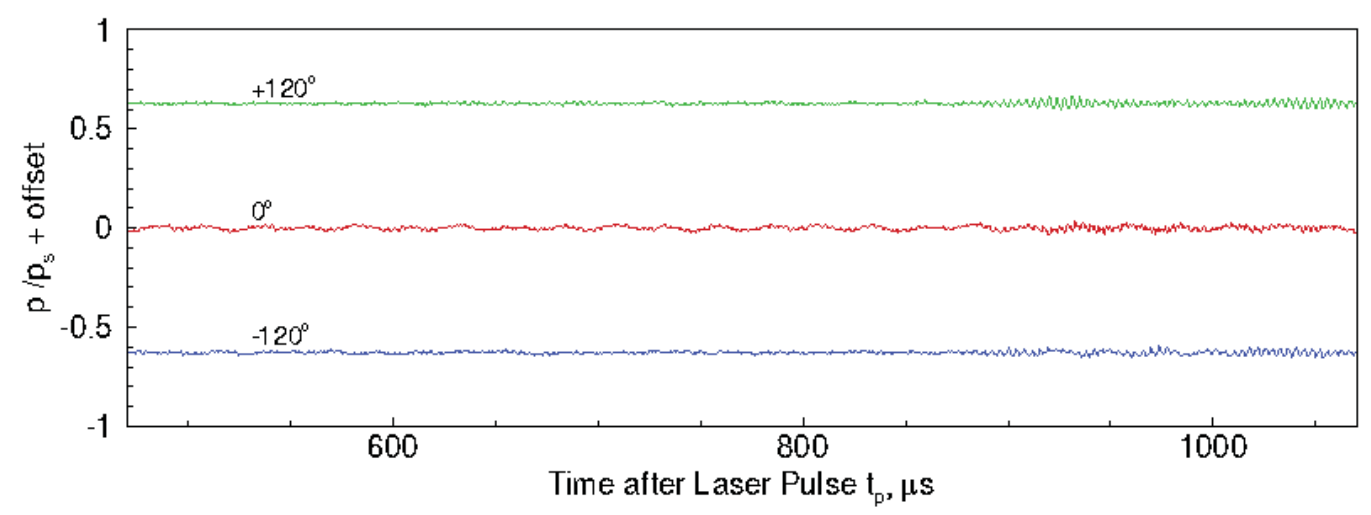

(a) $x=302 \mathrm{~mm}$.

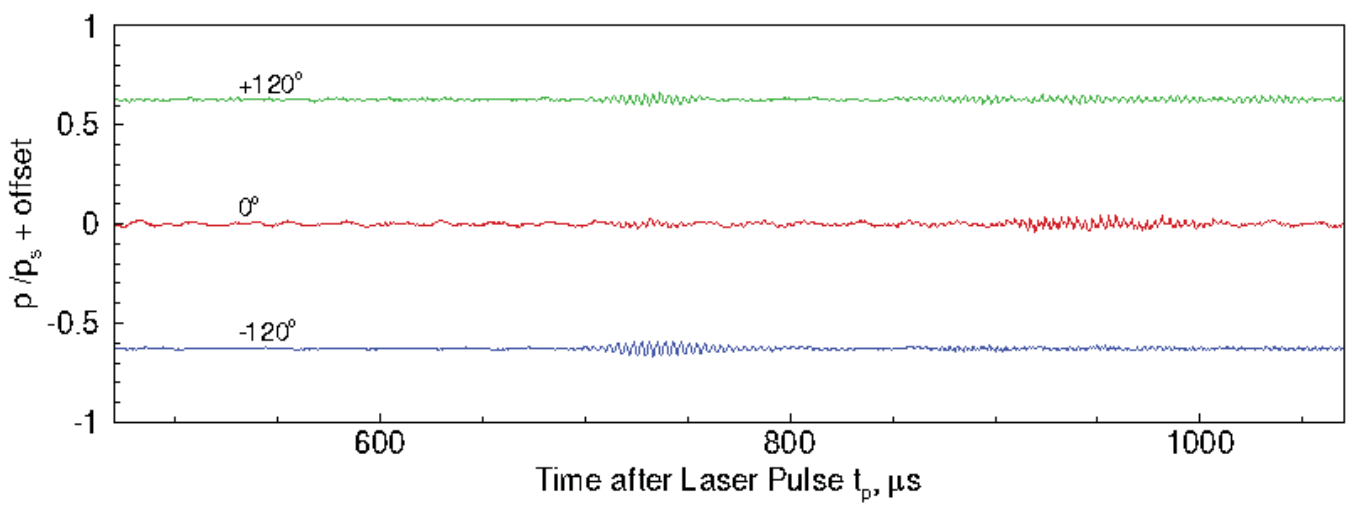

(b) $x=351 \mathrm{~mm}$.

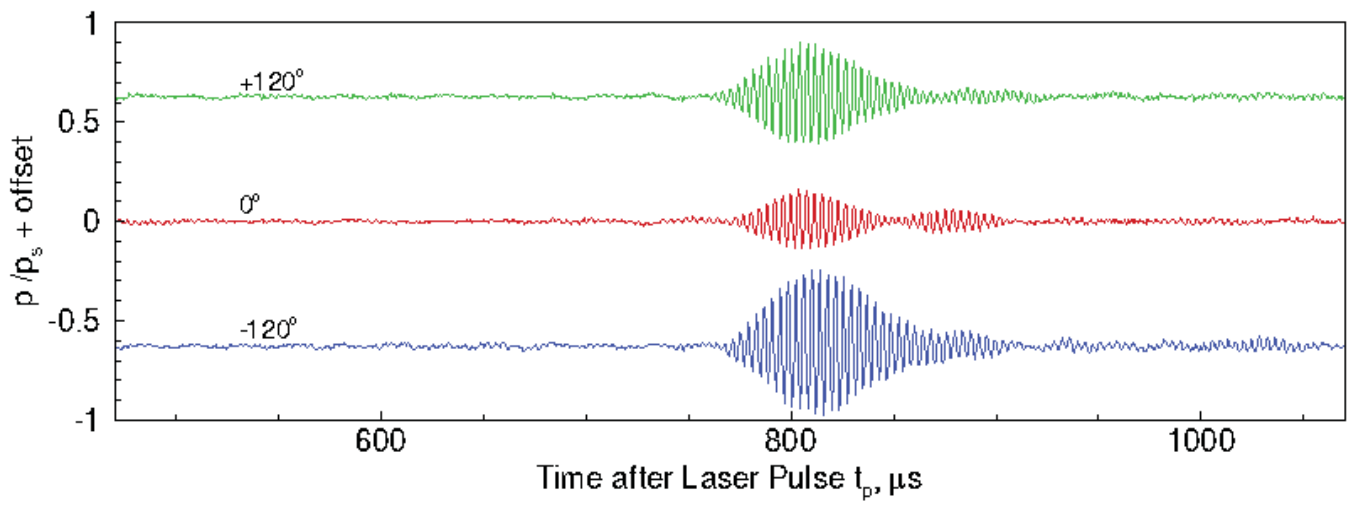

(c) $x=403 \mathrm{~mm}$

Figure 14. Measured time response to freestream laser perturbation on the blunt nosetip: $r_{n}=1 \mathrm{~mm} . p_{0}=$ $748.1 \mathrm{kPa}, T_{0}=431.9 \mathrm{~K}, \mathrm{Re} / \mathrm{m}=8.02 \times 10^{6} / \mathbf{m}$. Perturbation is aligned to cone centerline. 


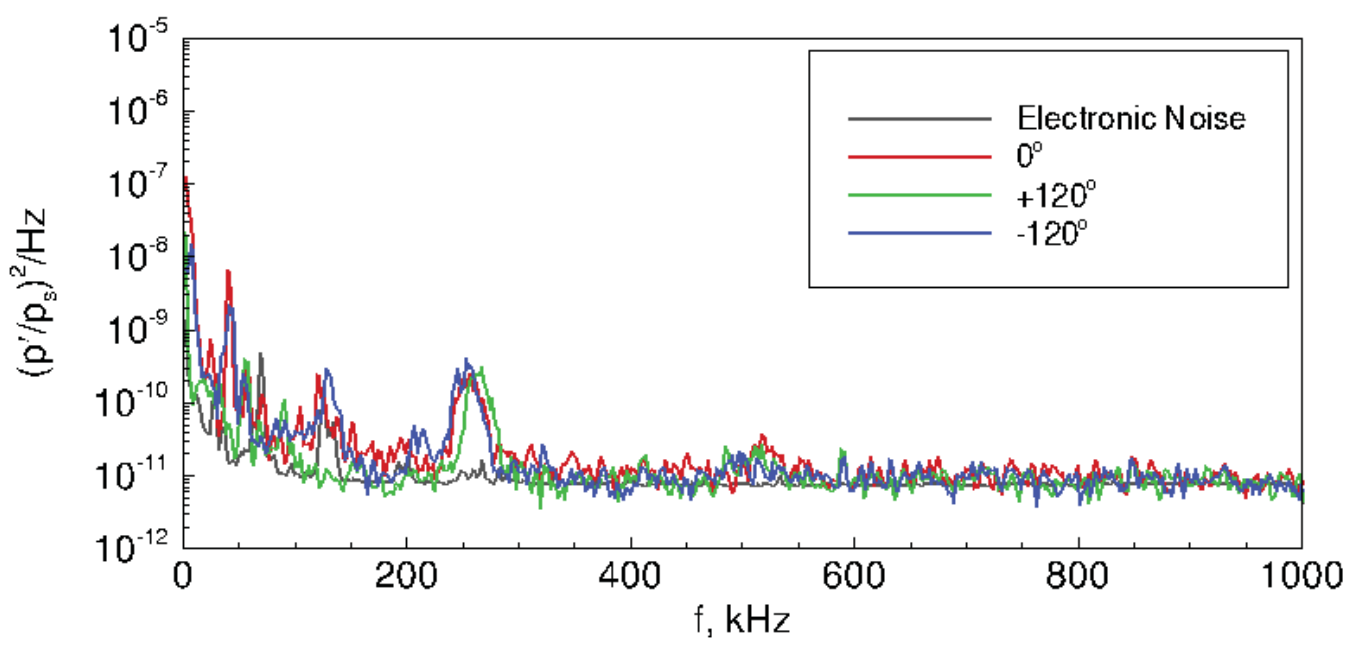

(a) $x=302 \mathrm{~mm}$.

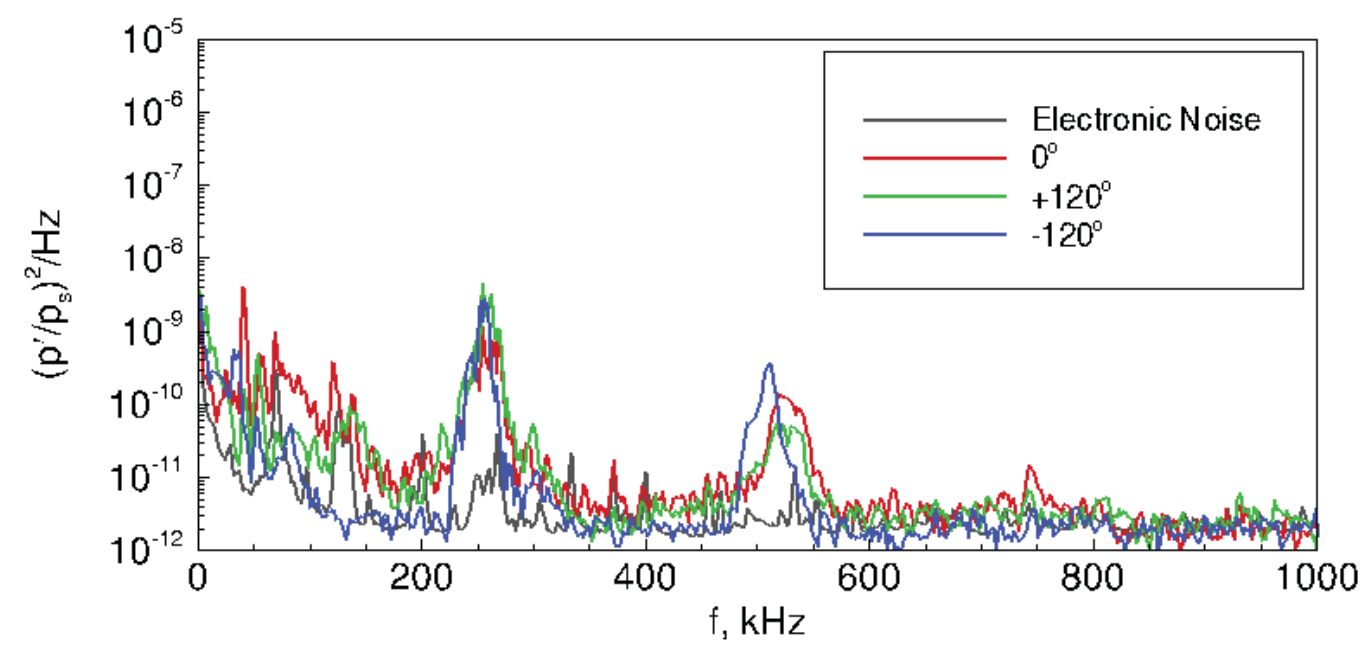

(b) $x=351 \mathrm{~mm}$.

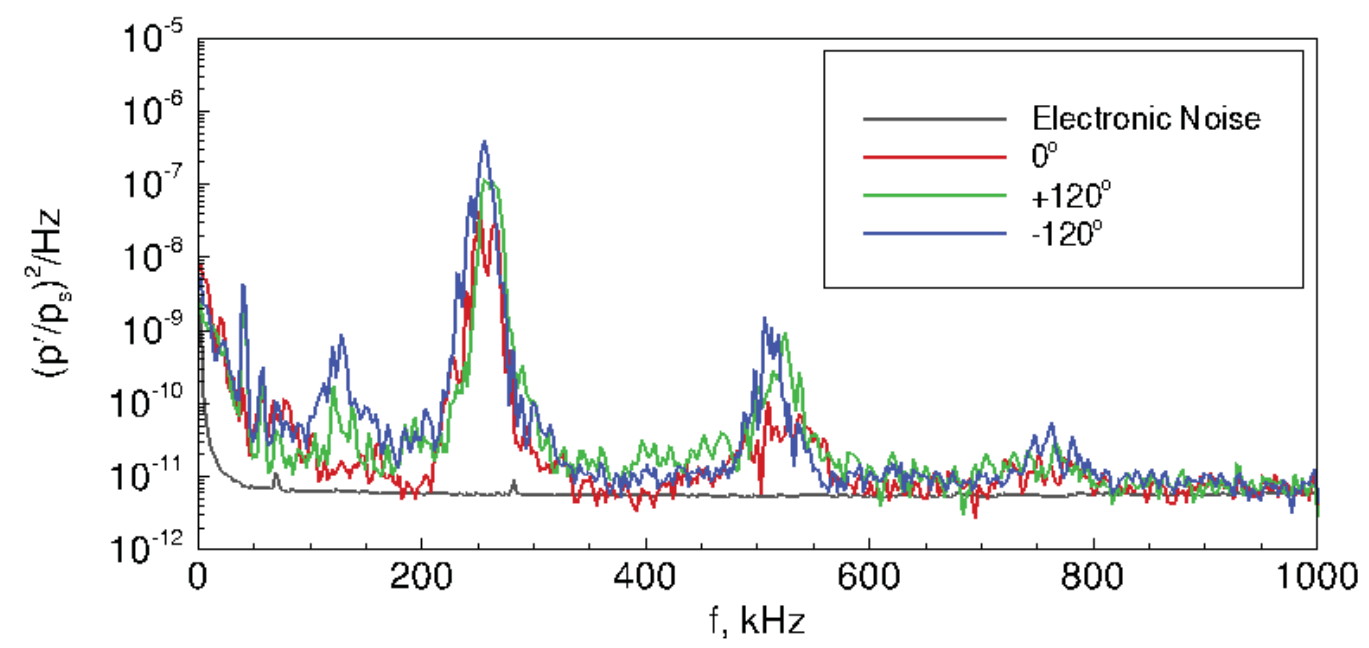

(c) $x=403 \mathrm{~mm}$.

Figure 15. Power spectral density of measurements on the blunt nosetip: $r_{n}=1 \mathbf{m m} . p_{0}=748.1 \mathrm{kPa}, T_{0}=431.9 \mathrm{~K}$, $R e / m=8.02 \times 10^{6} / \mathbf{m}$. Perturbation is aligned to cone centerline. 


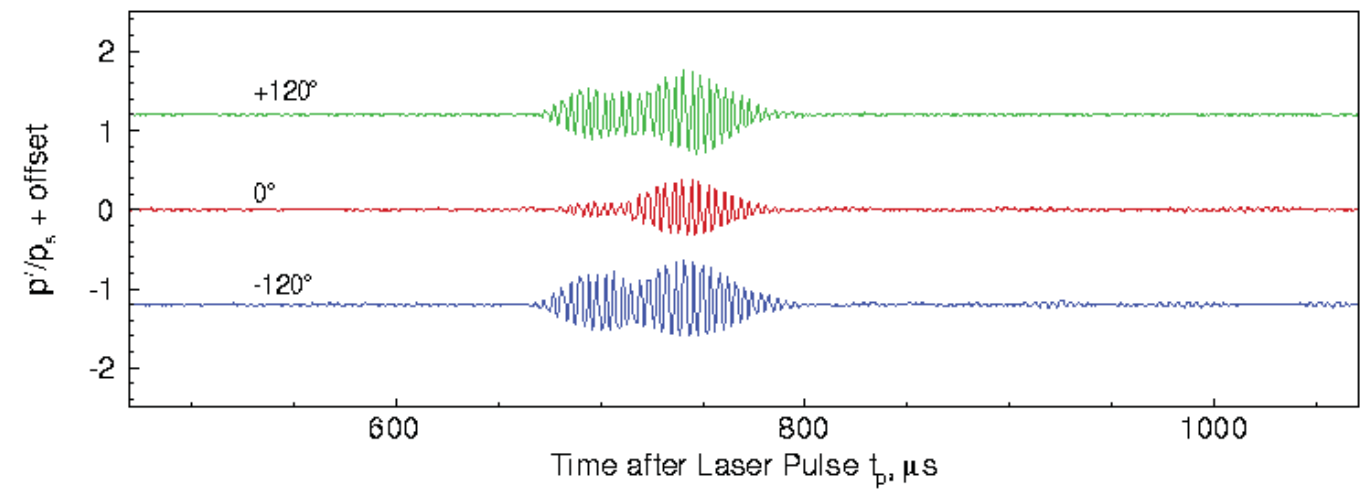

(a) Time response at $x=332 \mathrm{~mm}$.

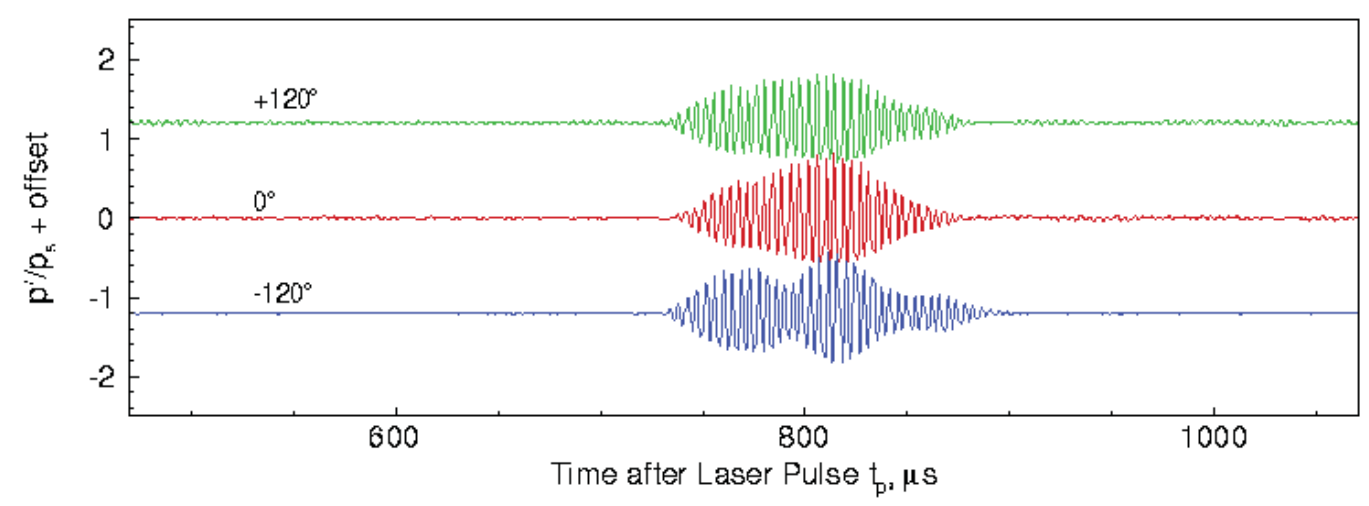

(b) Time response at $x=382 \mathrm{~mm}$.

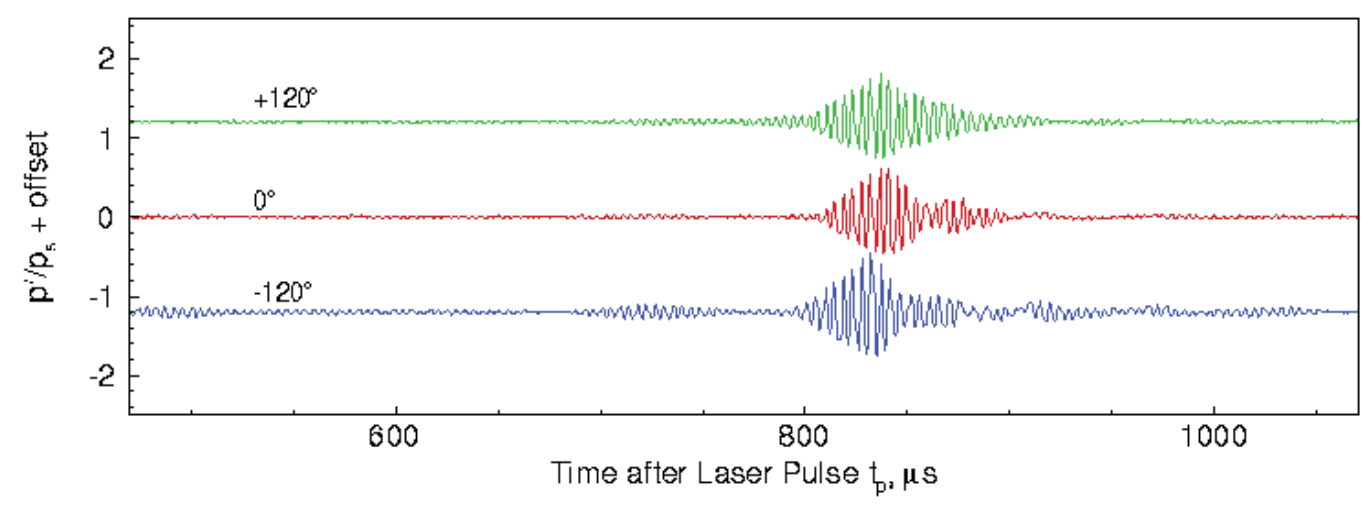

(c) Time response at $x=434 \mathrm{~mm}$.

Figure 16. Measured time response to freestream laser perturbation on the sharp nosetip: $r_{n}=0.16 \mathrm{~mm}$. $p_{0}=535.8 \mathrm{kPa}, T_{0}=424.1 \mathrm{~K}, \operatorname{Re} / m=5.92 \times 10^{6} / \mathbf{m}$. Perturbation is aligned to cone centerline. 


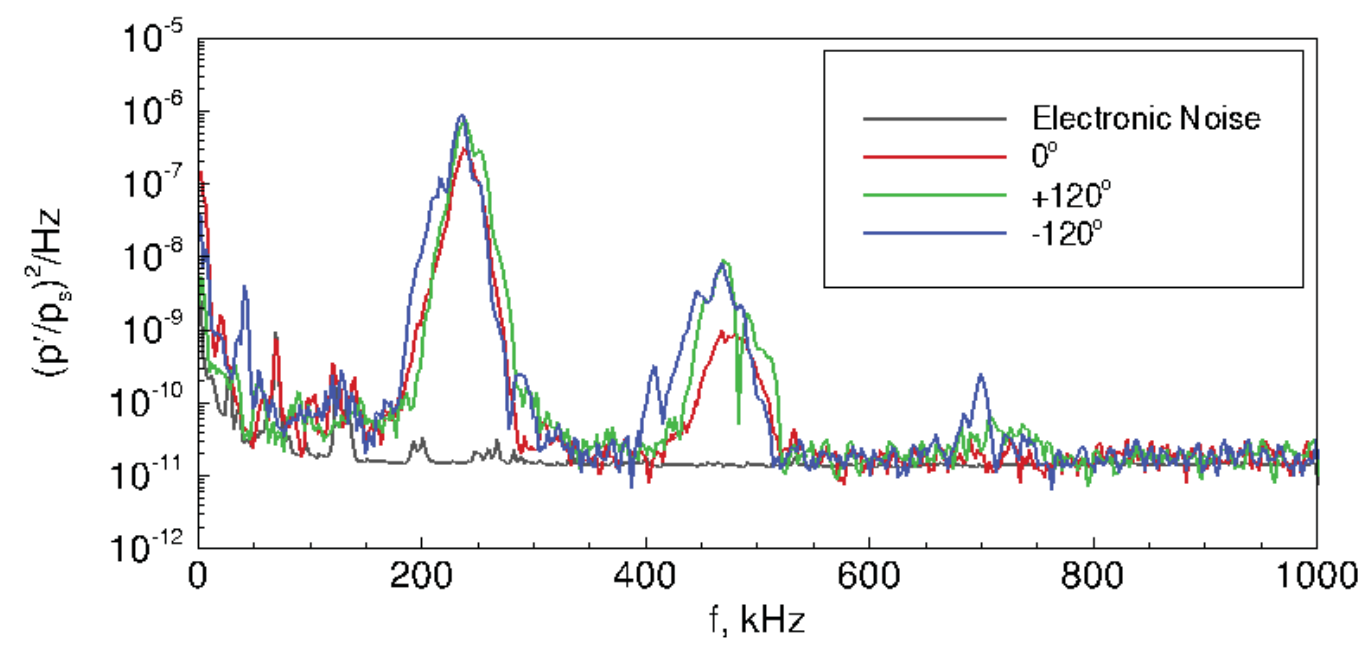

(a) Power spectral density at $x=332 \mathrm{~mm}$.

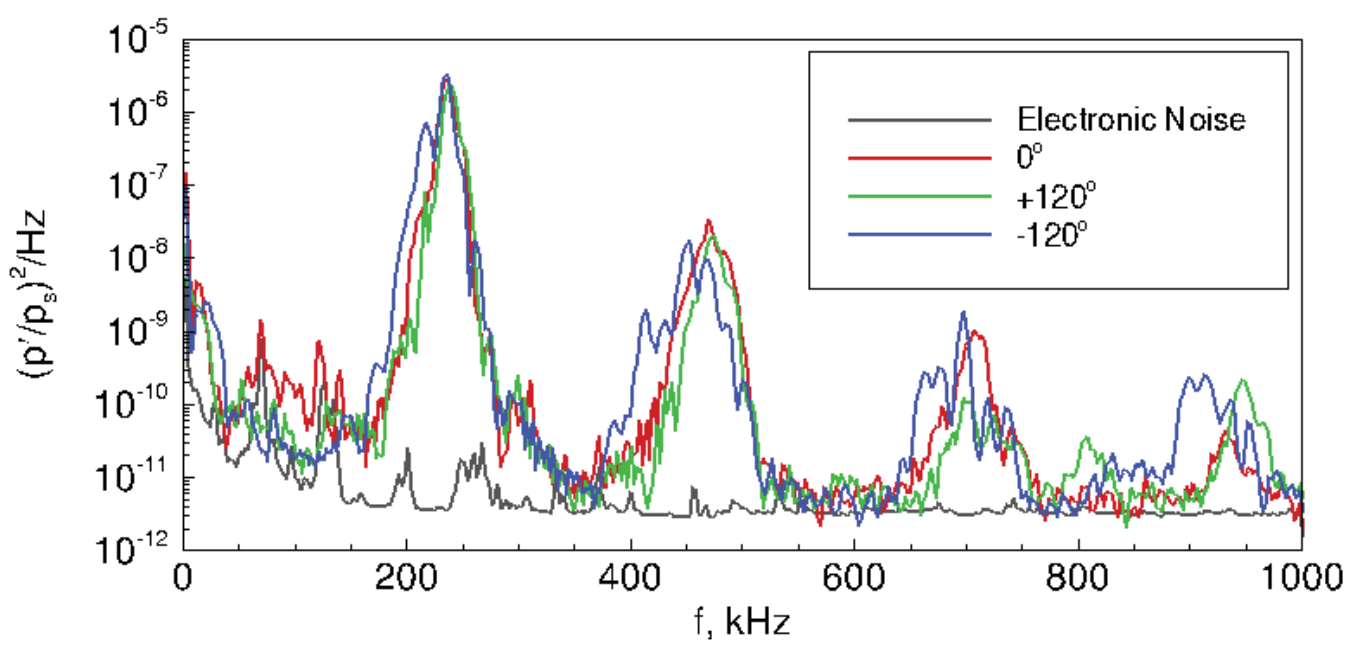

(b) Power spectral density at $x=382 \mathrm{~mm}$.

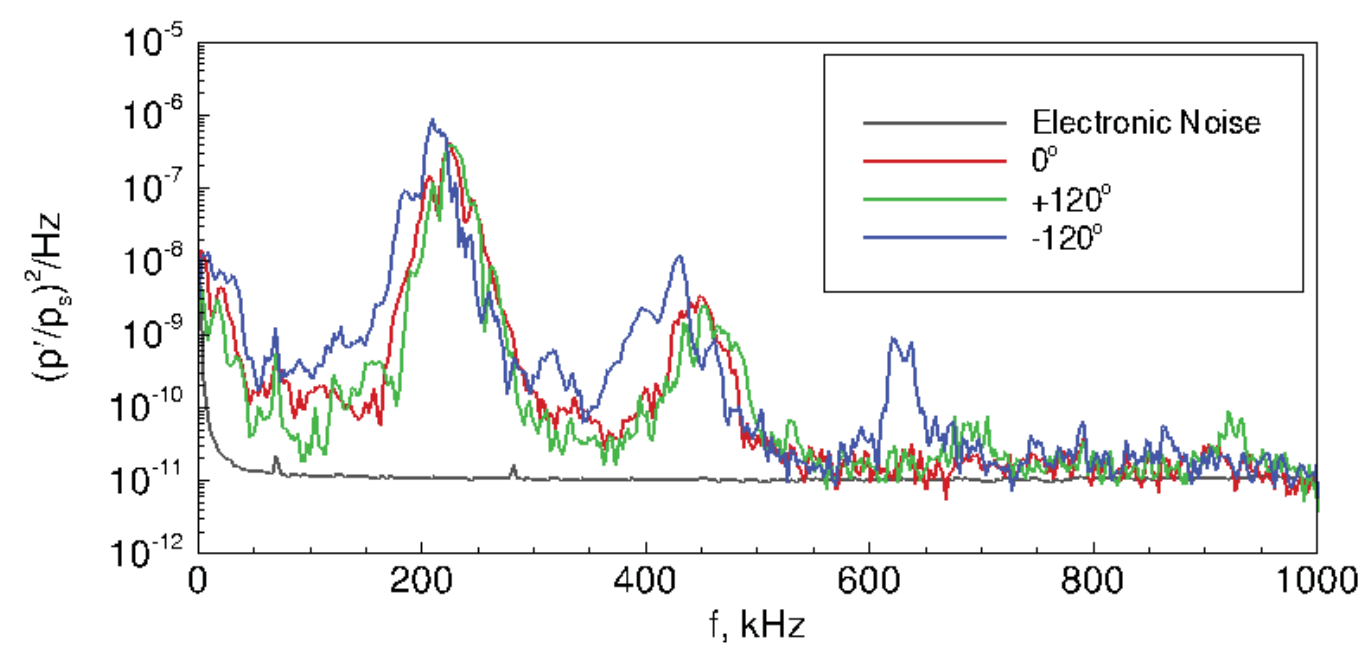

(c) Power spectral density at $x=434 \mathrm{~mm}$.

Figure 17. Power spectral density of measurements on the sharp nosetip: $r_{n}=0.16 \mathbf{m m} . p_{0}=535.8 \mathrm{kPa}$, $T_{0}=424.1 \mathrm{~K}, R e / m=5.92 \times 10^{6} / \mathbf{m}$. Perturbation is aligned to cone centerline. 
on the blunt flared cone. A peak at the expected second-mode frequency appears in the power spectra in Fig. 19(a). Again, an unknown slight increase in amplitude is seen at about $850 \mu$ s after the laser pulse is fired, despite the different setup. At the next azimuthal sensor array at $x=351 \mathrm{~mm}$, the wave packet on the $0^{\circ}$ ray appears to be significantly larger than the wave packets on the $+120^{\circ}$ and $-120^{\circ}$ rays. The power spectra in Fig. 19(b) indicate that the power in the second-mode instability is about an order of magnitude higher on the $0^{\circ}$ rays than the other two rays. Finally, in the most downstream of the azimuthal arrays, the wave packet on the $0^{\circ}$ ray is very large, while the wave packets on the $+120^{\circ}$ and $-120^{\circ}$ rays are similar in magnitude. The power spectra in Fig. 19(c) show that the power in the second-mode peak on the $0^{\circ}$ ray is almost 1.5 orders of magnitude larger than on the other two rays. However, the power spectra in Fig. 19(c) do not indicate that the wave packet has begun to break down on the $0^{\circ}$ ray. There are still clear peaks at the second-mode frequency band and at the first and second harmonics. The background frequency content remains close to the electronic noise floor. Similar effects were seen when the perturbation was offset toward either the $+120^{\circ}$ or $-120^{\circ}$ ray.

Figures 20 and 21 show the effect of the offset perturbation on the sharp flared cone. Surprisingly, the sharp nosetip does not appear to experience a change in amplitude around the azimuth like the blunt flared cone. Figure 20 shows the time traces from the three azimuthal sensor arrays. The shape of the packets appears to be fairly similar around the azimuth and the amplitude of the wave packets are similar. The power spectra in Fig. 21 all show that the second-mode frequency band is large and the first and second harmonics are present. This indicates that the wave packets are nonlinearly saturated on the sharp flared cone, and again, that small changes in the amplitude may be less evident. The power spectra in Fig. 21(c) show an increase in power in the lower frequencies on all three sensors at $x=434 \mathrm{~mm}$. This increase is probably due to the breakdown of the wave packet.

When the freestream perturbation was offset by as much as $3.0 \mathrm{~mm}$ from the centerline, the effect was more pronounced than when the offset was $1.5 \mathrm{~mm}$. Figure 22 shows the wave packet at three different azimuthal arrays on the blunt flared cone. On the blunt flared cone, the wave packet on the $0^{\circ}$ ray is similar to a turbulent spot. This is the ray toward which the freestream perturbation is offset. The other sensors on the $+120^{\circ}$ and $-120^{\circ}$ rays show no sign of the wave packet at $x=302 \mathrm{~mm}$ (Fig. 22(a)) and a small wave packet at $x=351 \mathrm{~mm}$ and $403 \mathrm{~mm}$ (Figs. 22(b) and 22(c)). The power spectra show that there is a large amount of power in the broadband frequency content for each sensor along the $0^{\circ}$ ray (Fig. 23). Thus, the wave packet appears to have broken down on the $0^{\circ}$ ray at even $x=302 \mathrm{~mm}$. The power spectra for the sensors on the $+120^{\circ}$ and $-120^{\circ}$ rays show the second-mode peak and some harmonics with relatively low background frequency content. Measurements made with the perturbation offset toward the $+120^{\circ}$ or $-120^{\circ}$ rays show similar results.

Figures 24 and 25 show the wave packet on three different azimuthal arrays on the sharp flared cone. The perturbation is offset from the cone centerline by $3 \mathrm{~mm}$ toward the $0^{\circ}$ ray. The wave packet on the sharp cone appears to be about the same shape and amplitude at each ray of sensors (Fig. 22), as in the previous cases. The power spectra also show relatively similar amplitudes in the second-mode frequency band and in the higher harmonics (Fig. 23). However, there appear to be signs that breakdown is starting to occur at $x=434 \mathrm{~mm}$ along the $0^{\circ}$ ray. A rise in the power of the lower frequencies between about $0-450 \mathrm{kHz}$ is shown in Fig. 23(c) for the sensor at $0^{\circ}$. The content in this frequency band at $+120^{\circ}$ and $-120^{\circ}$ is much less than on the $0^{\circ}$ ray. The effect of the offset perturbation on the sharp flared cone is still less than the effect on the blunt flared cone. For both offsets used in this experiment, the sharp nosetip appears to be less sensitive to the alignment of the freestream perturbation than the blunt nosetip.

\section{Summary}

This experiment provides unique insight into the receptivity process. These measurements are the first to show that a controlled freestream perturbation can generate a wave packet on a model. This wave packet grows, becomes nonlinearly saturated, and then experiences breakdown. While the measurements may not be as complete or as accurate as desired, they are necessarily so due to the difficulty in making them.

These measurements show that a small boundary layer instability can be generated by a large, discrete thermal perturbation in the freestream. The freestream laser-generated perturbation creates a wave packet with the same frequency content as a natural instability wave in the boundary layer. When the perturbation is offset from the cone centerline, a larger wave packet is generated on the side of the blunt flared cone toward which the perturbation is offset. Similar effects of the offset perturbation are not observed on the 


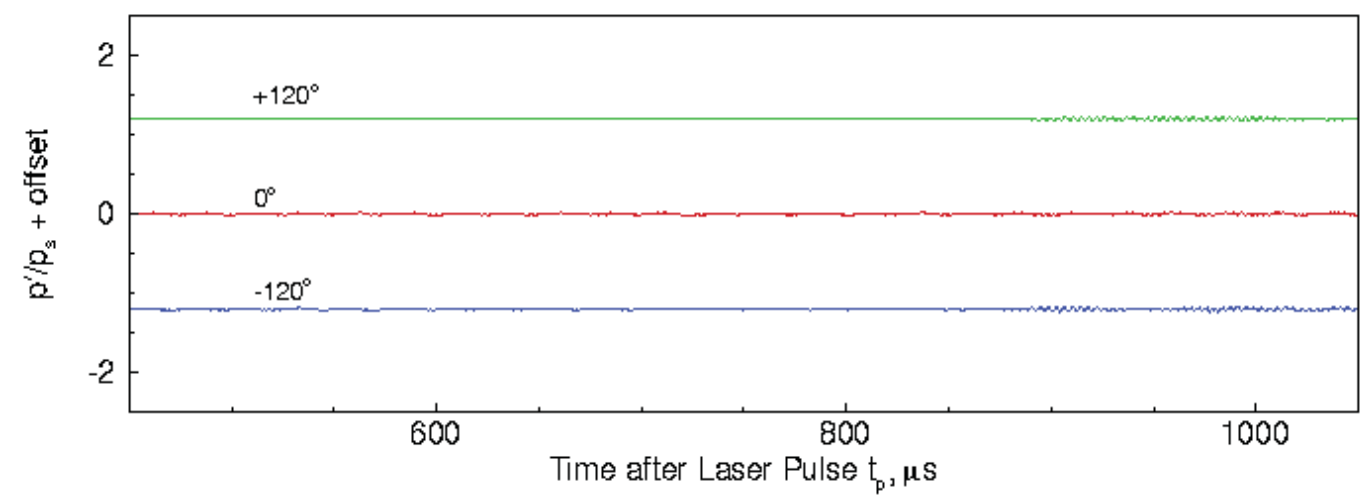

(a) $x=302 \mathrm{~mm}$.

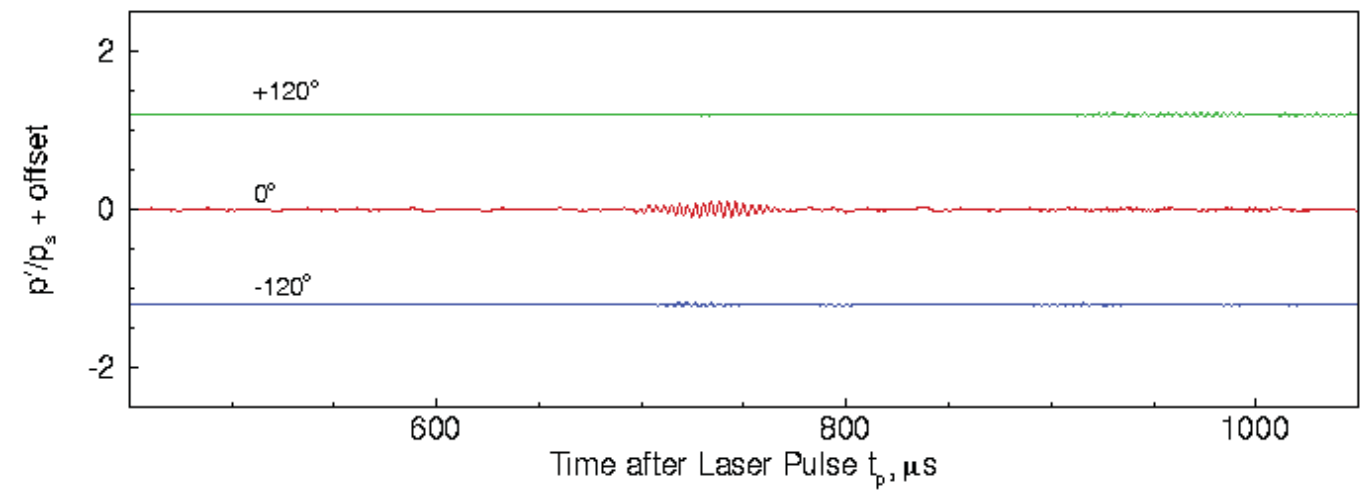

(b) $x=351 \mathrm{~mm}$.

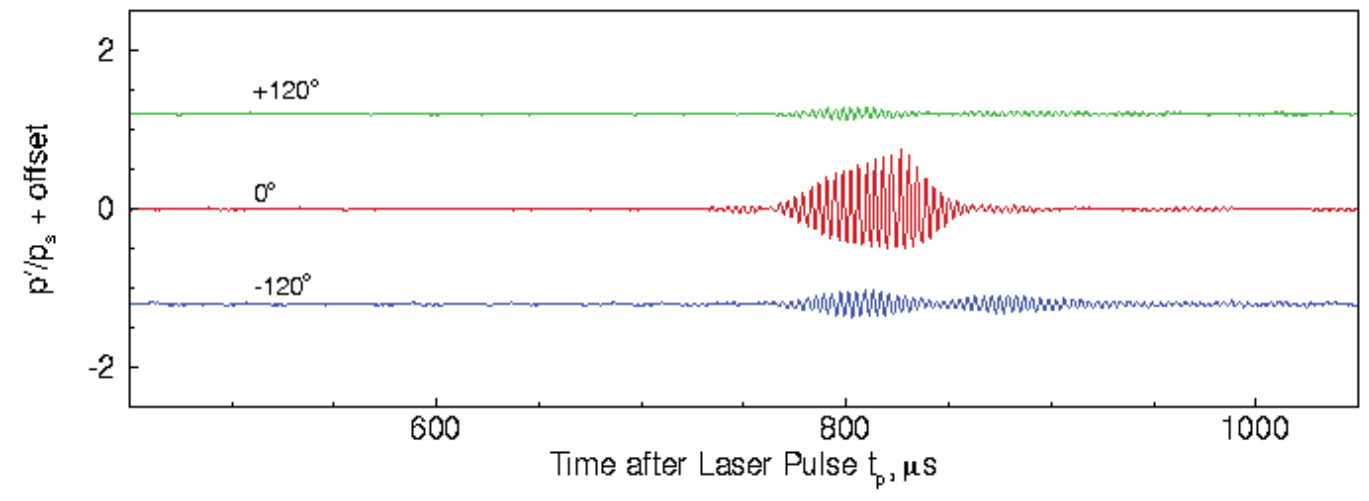

(c) $x=403 \mathrm{~mm}$.

Figure 18. Measured time response to freestream laser perturbation on the blunt nosetip: $r_{n}=1 \mathrm{~mm} . p_{0}=$ $734.6 \mathrm{kPa}, T_{0}=429.9 \mathrm{~K}, R e / m=7.93 \times 10^{6} / \mathbf{m}$. Perturbation is offset $1.5 \mathrm{~mm}$ from the cone centerline toward the $0^{\circ}$ ray. 


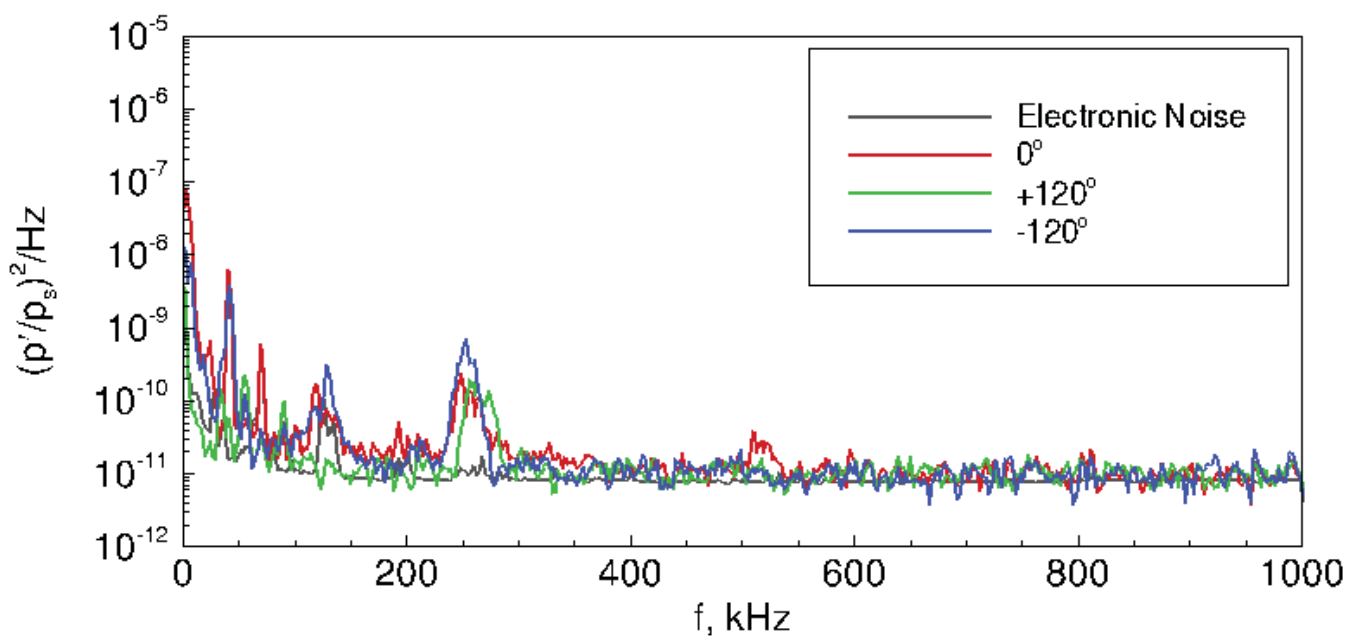

(a) $x=302 \mathrm{~mm}$.

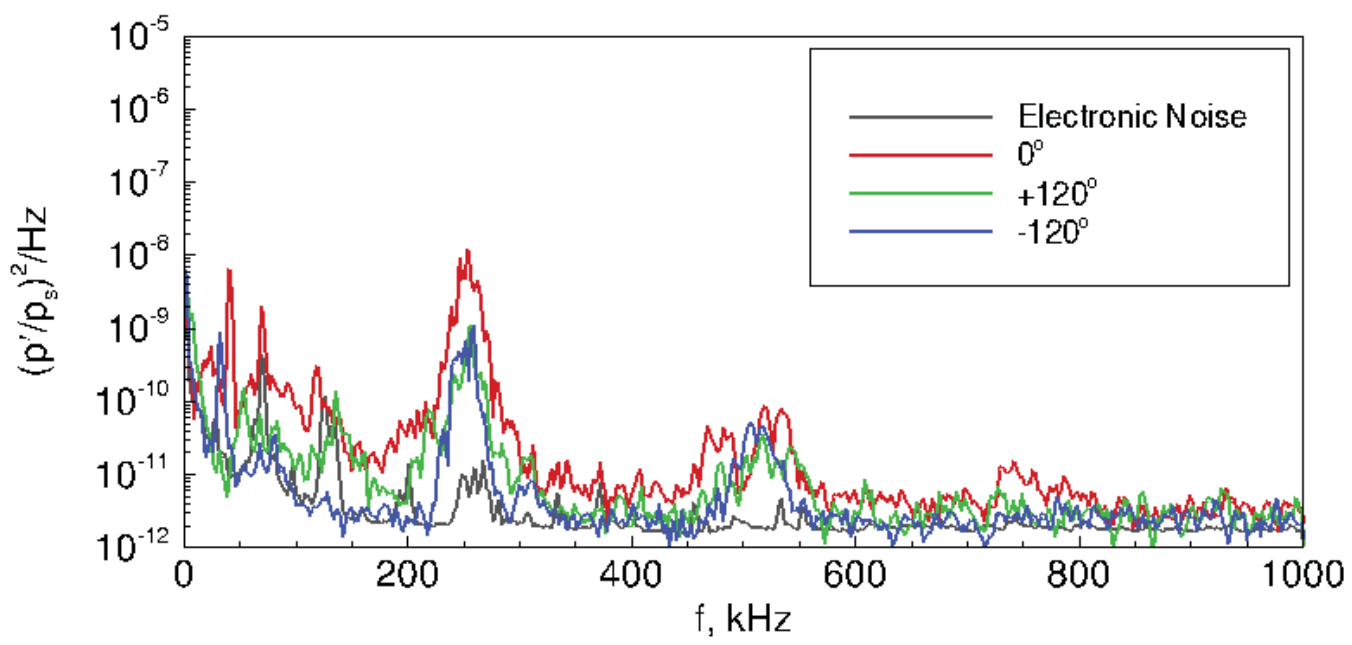

(b) $x=351 \mathrm{~mm}$.

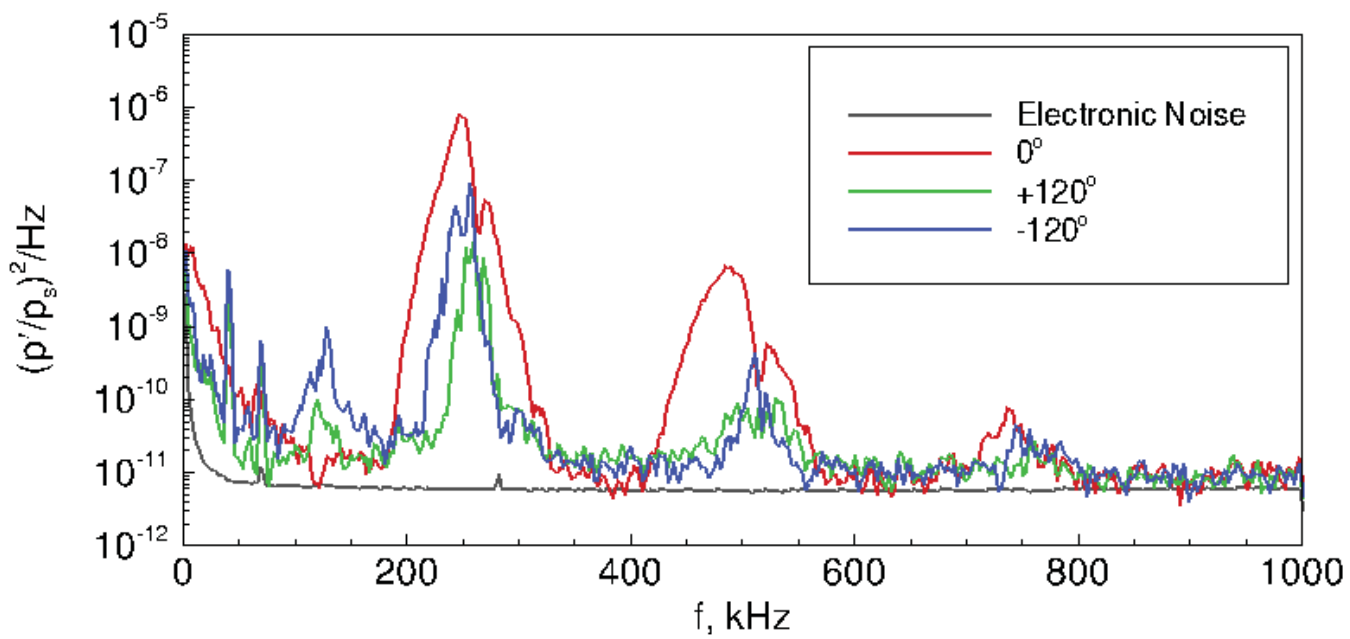

(c) $x=403 \mathrm{~mm}$.

Figure 19. Power spectral density of measurements on the blunt nosetip: $r_{n}=1 \mathbf{m m} . p_{0}=734.6 \mathbf{~ k P a}, T_{0}=429.9 \mathrm{~K}$, $R e / m=7.93 \times 10^{6} / \mathbf{m}$. Perturbation is offset $1.5 \mathbf{~ m m}$ from the cone centerline toward the $0^{\circ}$ ray. 


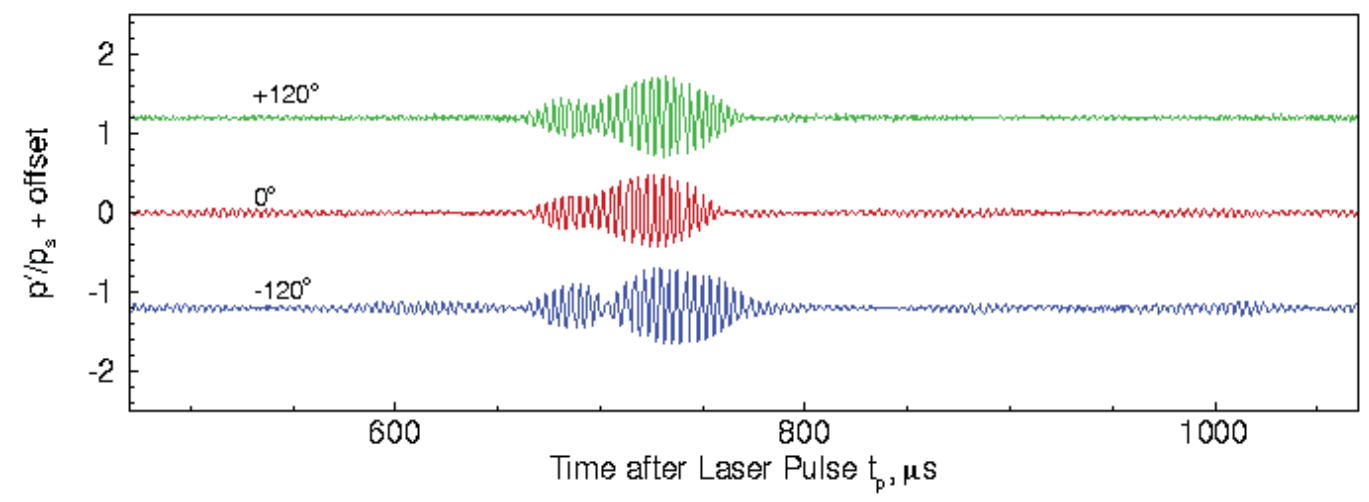

(a) $x=332 \mathrm{~mm}$.

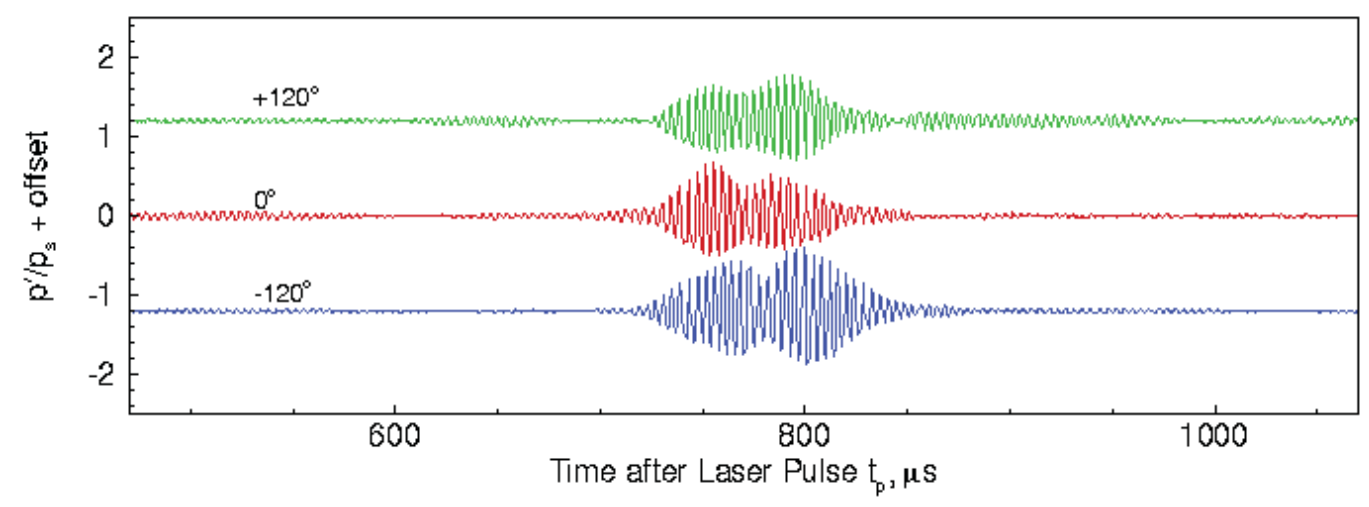

(b) $x=382 \mathrm{~mm}$.

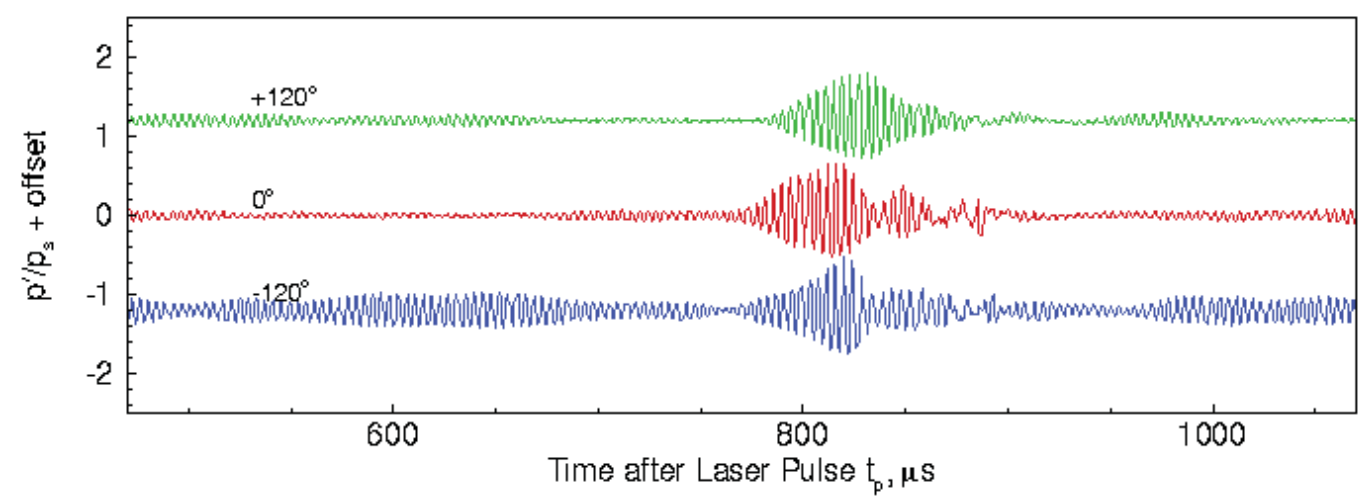

(c) $x=434 \mathrm{~mm}$.

Figure 20. Measured time response to freestream laser perturbation on the sharp nosetip: $r_{n}=0.16 \mathrm{~mm}$. $p_{0}=586.4 \mathrm{kPa}, T_{0}=427.4 \mathrm{~K}, \operatorname{Re} / m=6.40 \times 10^{6} / \mathbf{m}$. Perturbation is aligned to cone centerline. 


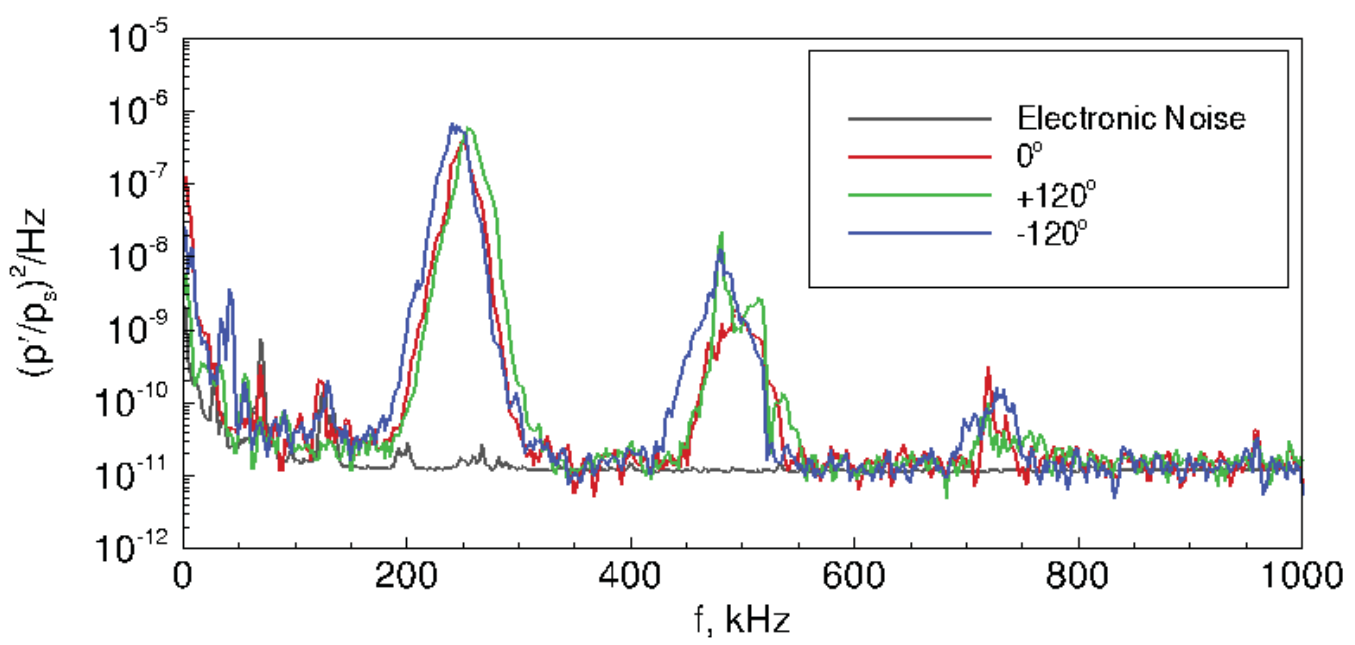

(a) $x=332 \mathrm{~mm}$.

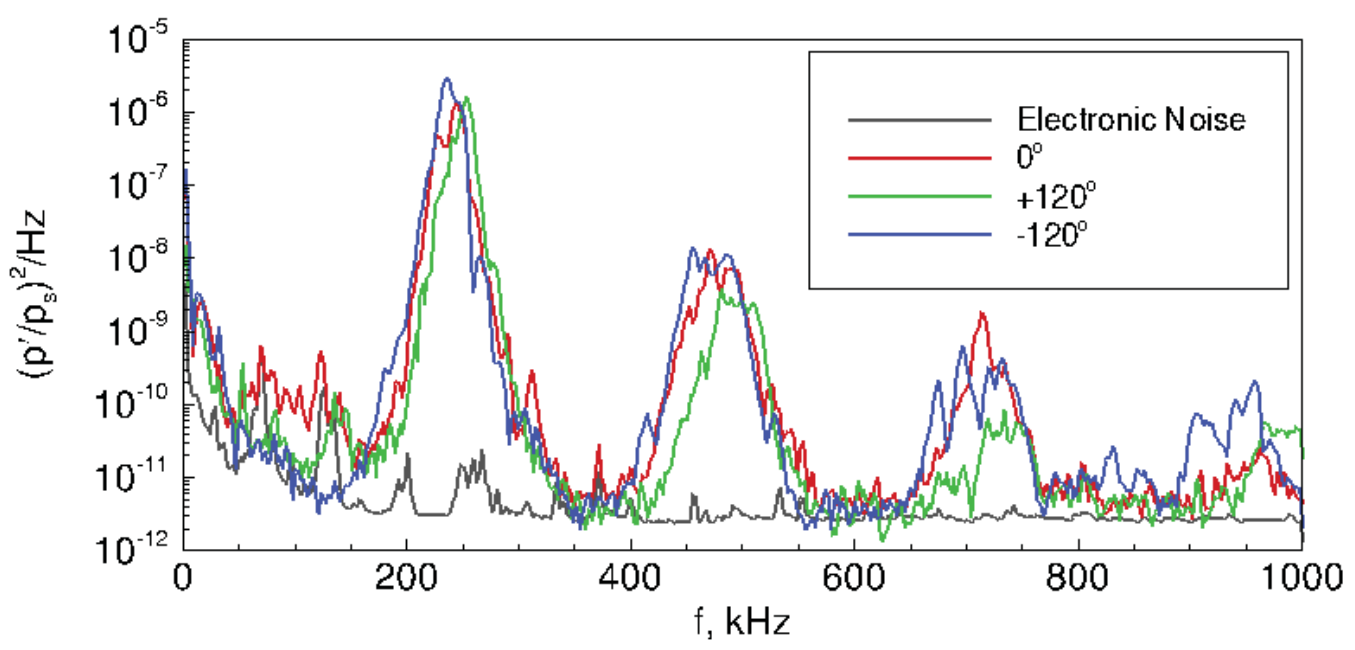

(b) $x=382 \mathrm{~mm}$.

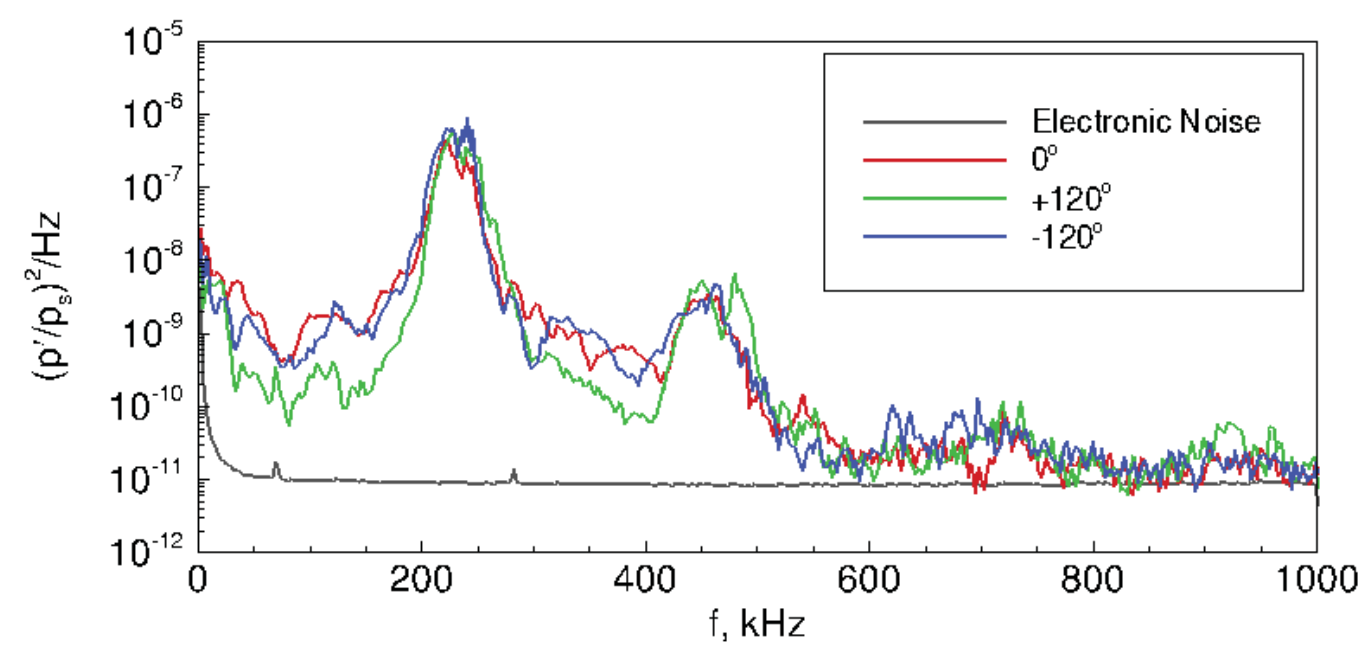

(c) $x=434 \mathrm{~mm}$.

Figure 21. Power spectral density of measurements on the sharp nosetip: $r_{n}=0.16 \mathrm{~mm} . p_{0}=586.4 \mathrm{kPa}$, $T_{0}=427.4 \mathrm{~K}, R e / m=6.40 \times 10^{6} / \mathbf{m}$. Perturbation is aligned to cone centerline. 


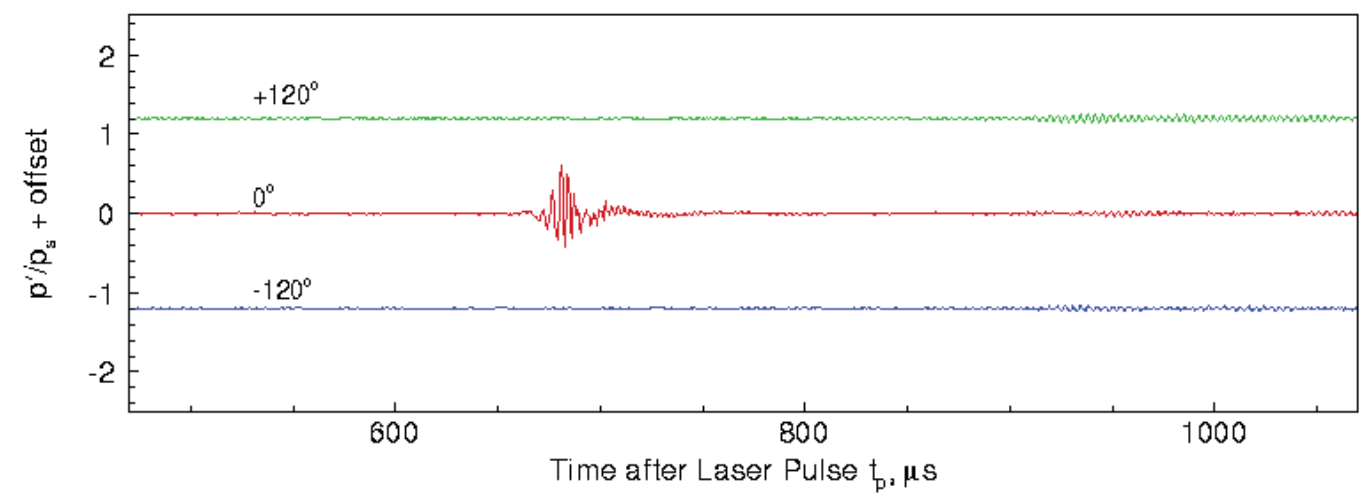

(a) $x=302 \mathrm{~mm}$.

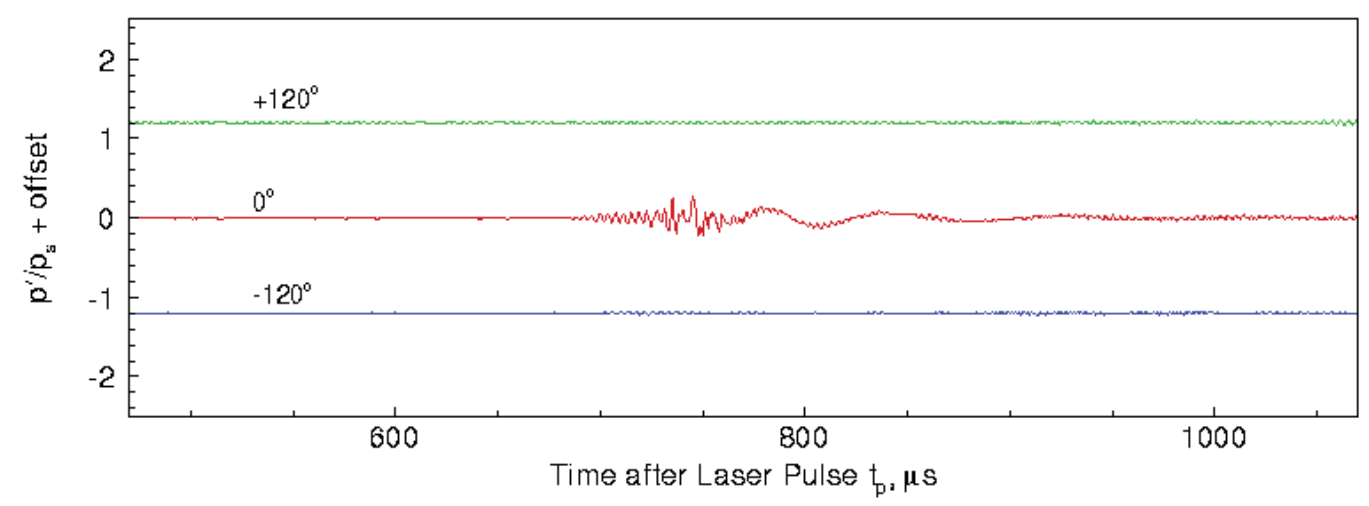

(b) $x=351 \mathrm{~mm}$.

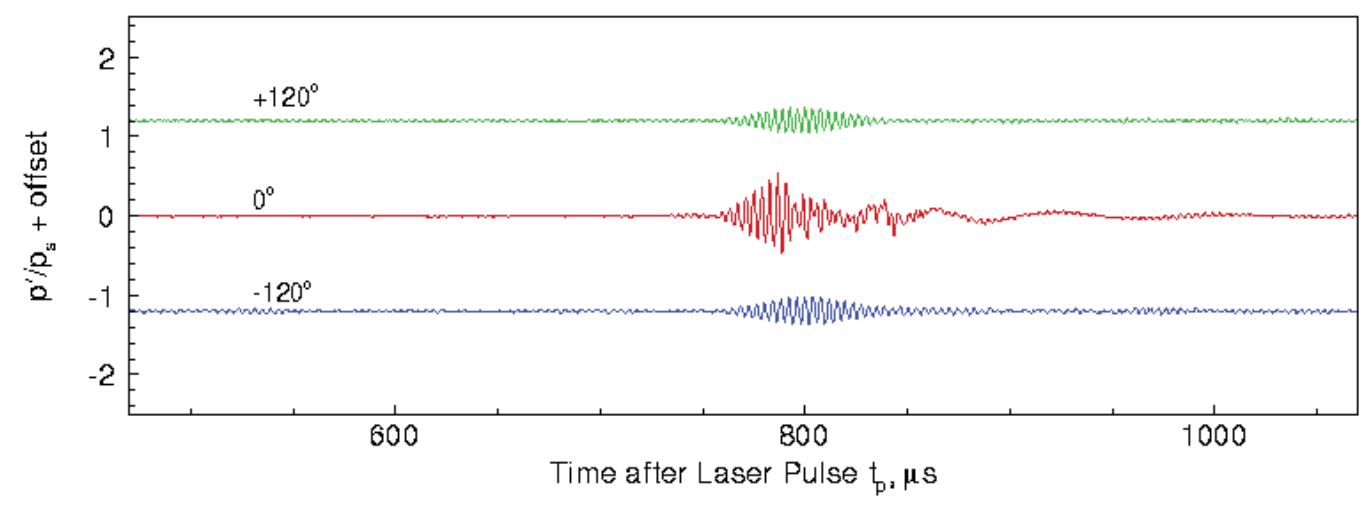

(c) $x=403 \mathrm{~mm}$.

Figure 22. Measured time response to freestream laser perturbation on the blunt nosetip: $r_{n}=1 \mathrm{~mm} . p_{0}=$ $749.9 \mathrm{kPa}, T_{0}=431.5 \mathrm{~K}, R e / m=8.05 \times 10^{6} / \mathbf{m}$. Perturbation is offset $3.0 \mathbf{~ m m}$ from centerline toward the $0^{\circ}$ ray. 


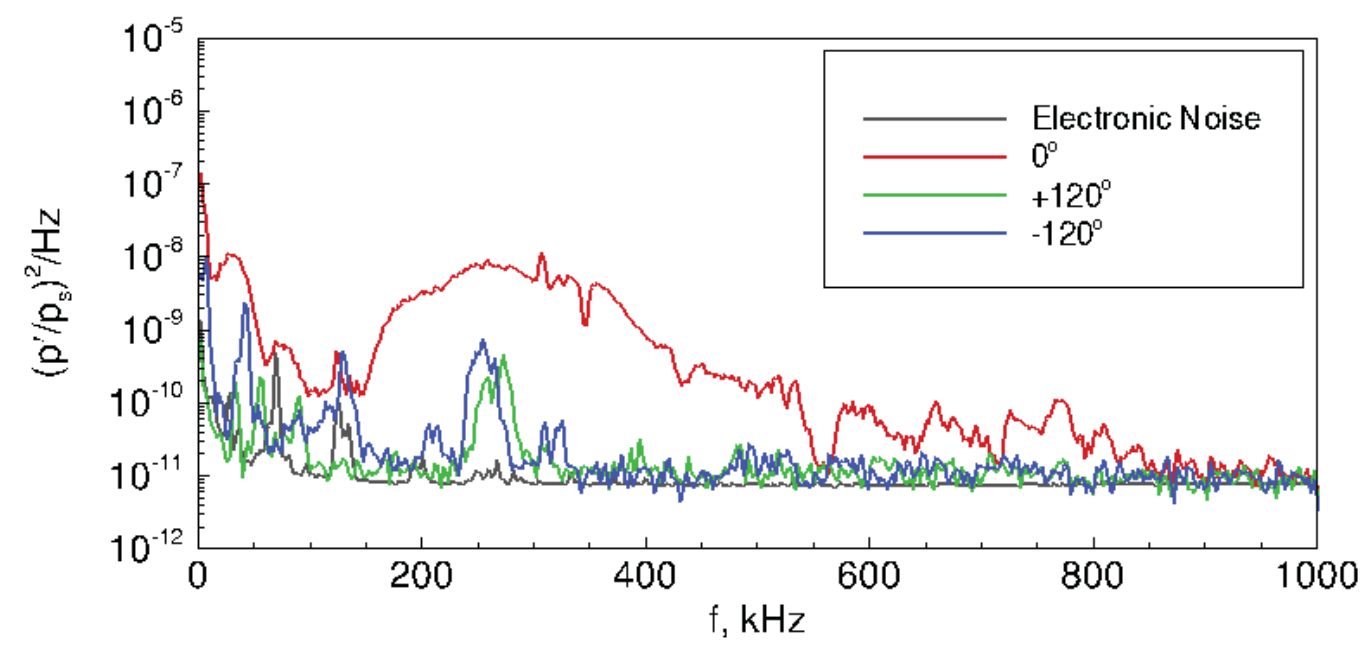

(a) $x=302 \mathrm{~mm}$.

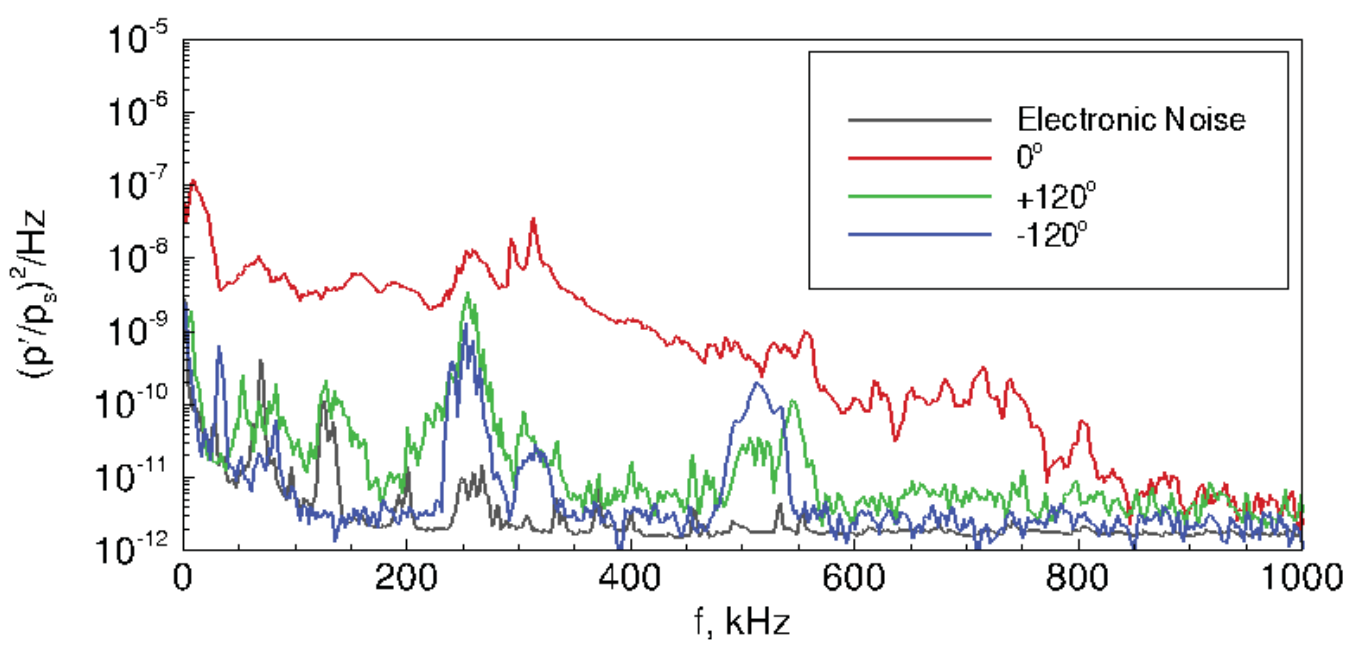

(b) $x=351 \mathrm{~mm}$.

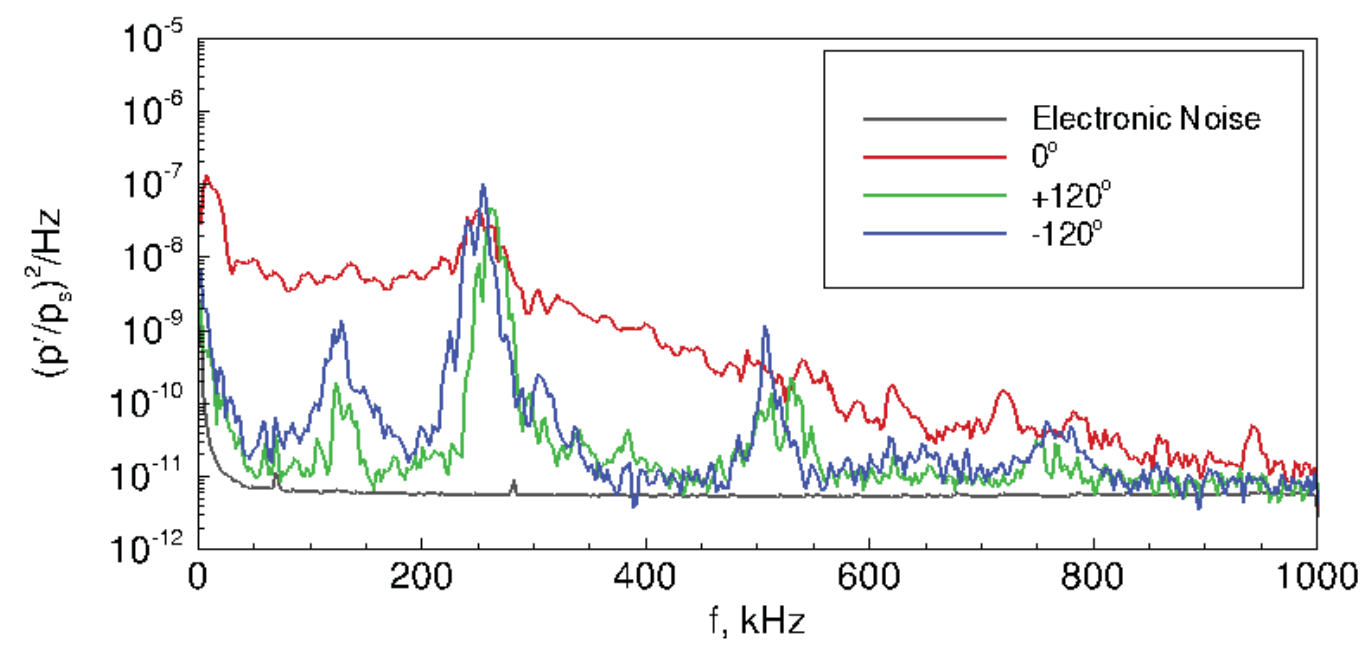

(c) $x=403 \mathrm{~mm}$.

Figure 23. Power spectral density of measurements on the blunt nosetip: $r_{n}=1 \mathbf{m m} . p_{0}=749.9 \mathrm{kPa}, T_{0}=431.5 \mathrm{~K}$, $R e / m=8.05 \times 10^{6} / \mathbf{m}$. Perturbation is offset $\mathbf{3 . 0} \mathbf{~ m m}$ from centerline toward the $\mathbf{0}^{\circ}$ ray. 


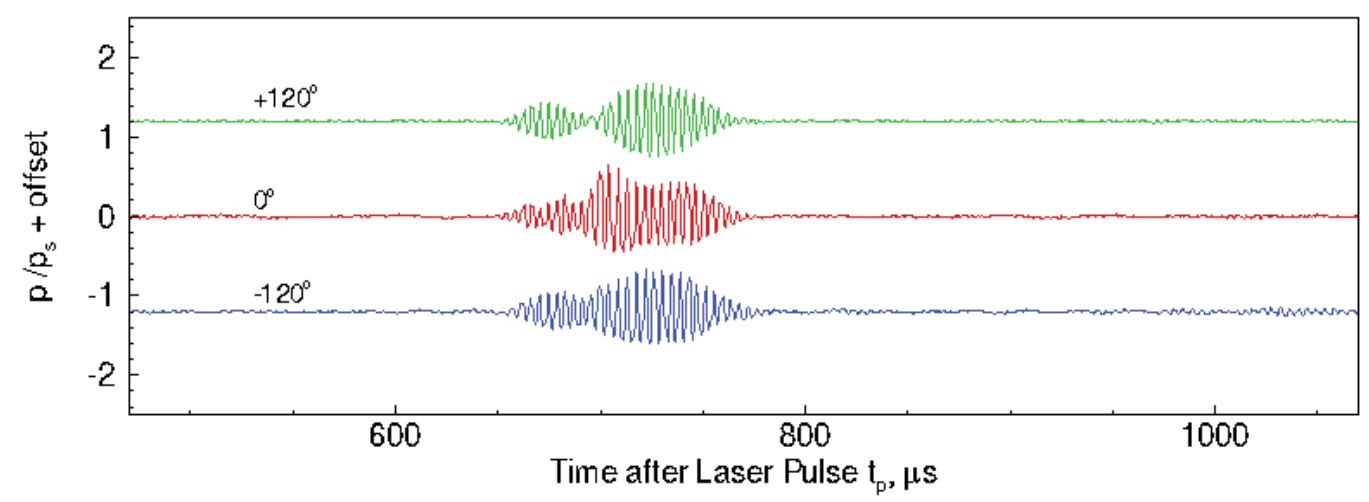

(a) $x=332 \mathrm{~mm}$.

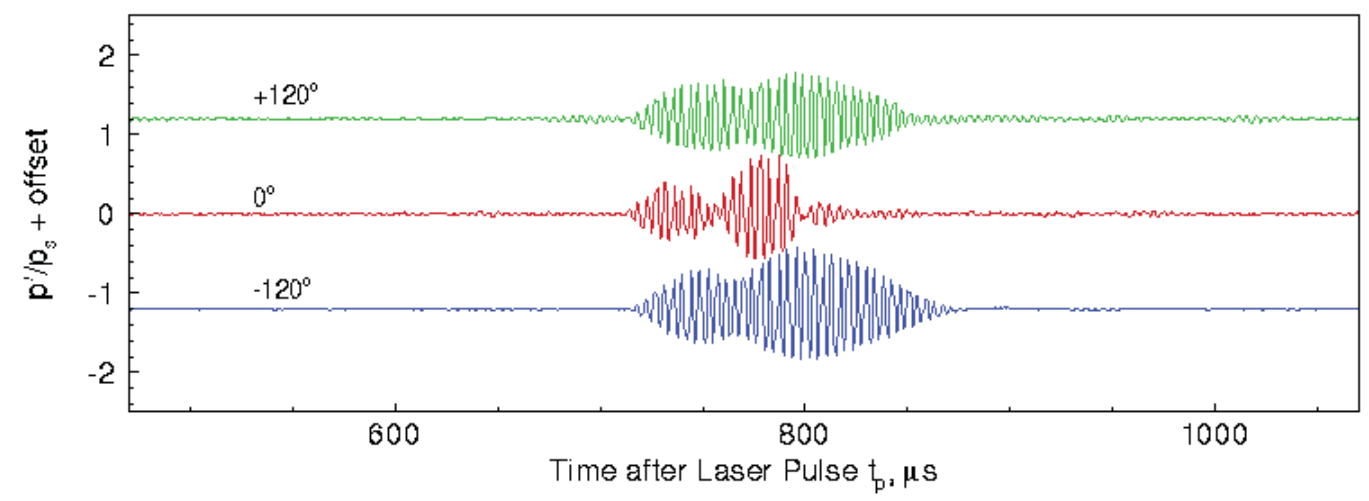

(b) $x=382 \mathrm{~mm}$.

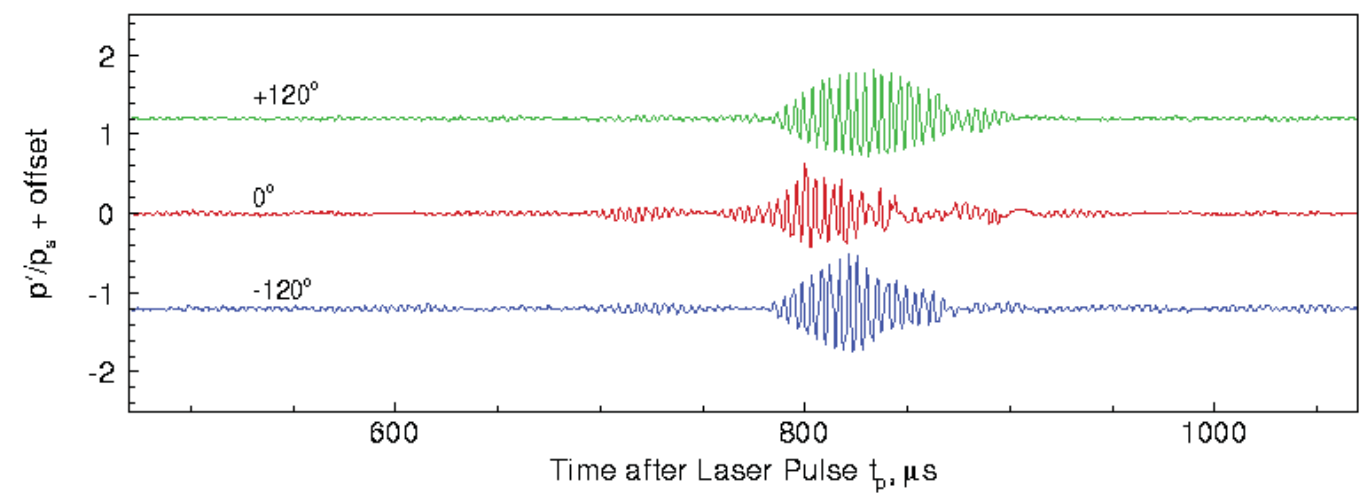

(c) $x=434 \mathrm{~mm}$.

Figure 24. Measured time response to freestream laser perturbation on the sharp nosetip: $r_{n}=0.16 \mathrm{~mm}$. $p_{0}=533.9 \mathrm{kPa}, T_{0}=427.5 \mathrm{~K}, \operatorname{Re} / m=5.82 \times 10^{6} / \mathbf{m}$. Perturbation is offset $3.0 \mathrm{~mm}$ from centerline toward the $0^{\circ}$ ray. 


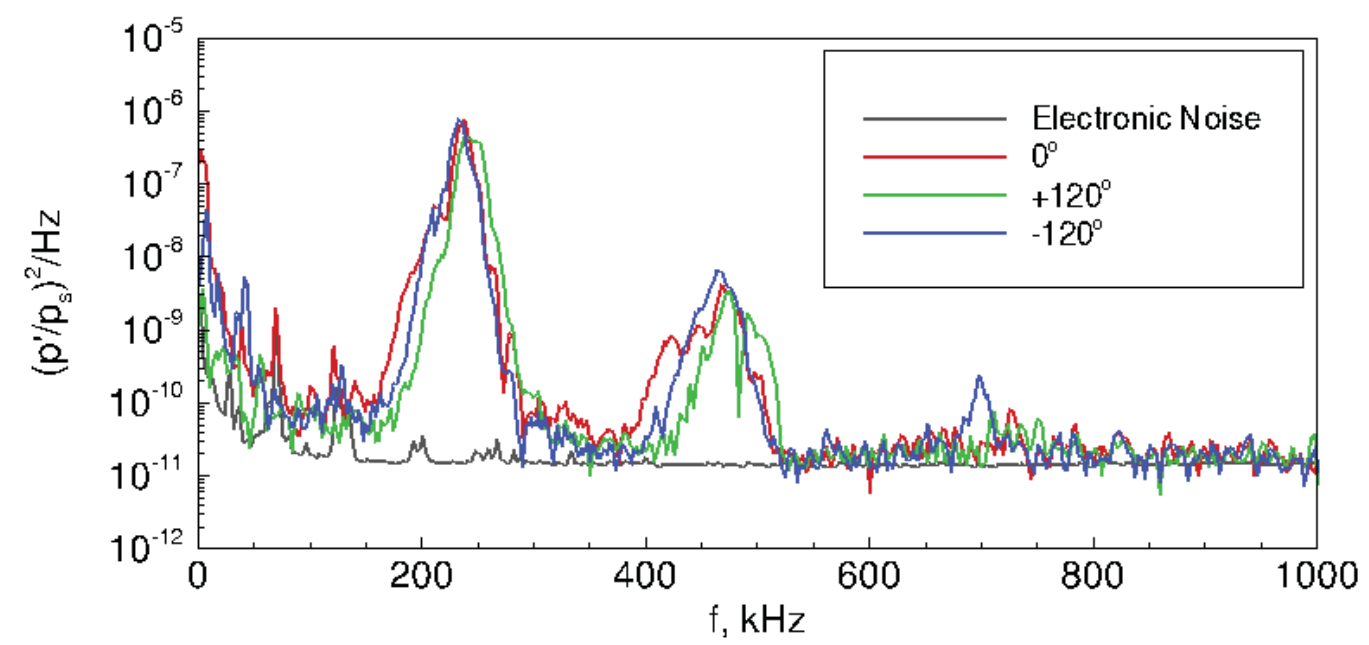

(a) $x=332 \mathrm{~mm}$.

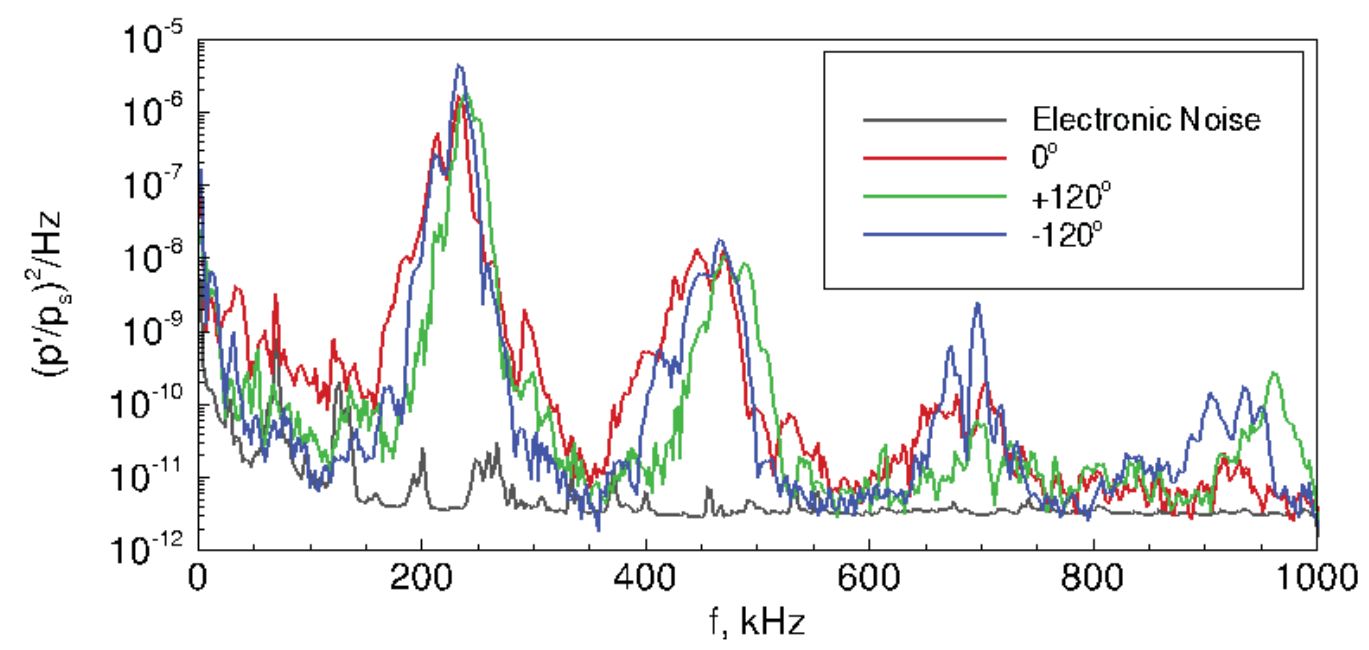

(b) $x=382 \mathrm{~mm}$.

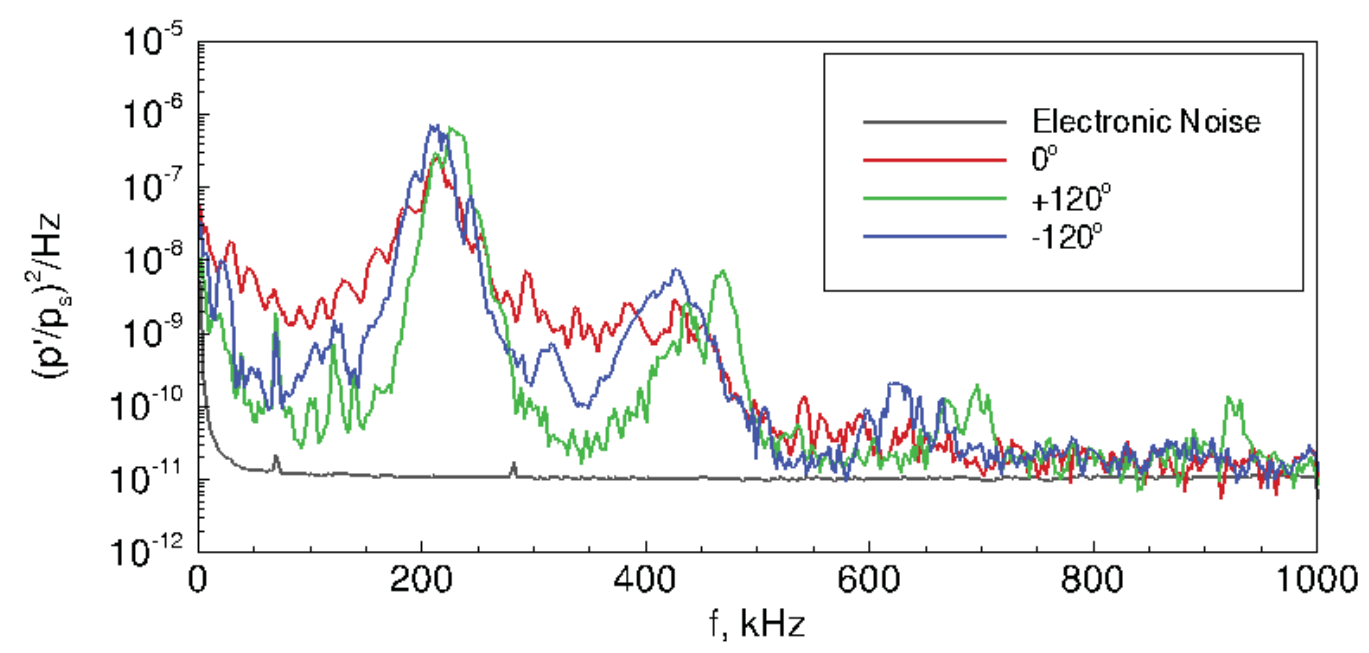

(c) $x=434 \mathrm{~mm}$.

Figure 25. Power spectral density of measurements on the sharp nosetip: $r_{n}=0.16 \mathrm{~mm} . p_{0}=533.9 \mathrm{kPa}$, $T_{0}=427.5 \mathrm{~K}, R e / m=5.82 \times 10^{6} / \mathbf{m}$. Perturbation is offset $3.0 \mathrm{~mm}$ from centerline toward the $0^{\circ}$ ray. 
sharp flared cone.

These data also raise questions about the receptivity process. Wave packets with small initial amplitudes were generated by a freestream thermal disturbance with a large peak magnitude. Prior to this study, the magnitude of the freestream disturbance was suspected to be too large or incapable of generating measurable wave packets. However, this freestream disturbance is of short duration, so it is possible that the integrated effect of the perturbation is small enough to cause linear receptivity. The effect of the offset perturbation on the wave packet amplitude is also unexpected. The offset perturbation has a larger effect on the blunt flared cone than on the sharp flared cone, but the reason for this difference is not known.

These questions which will take more research and analysis to resolve. Computations of the interaction between the flared cone and the large, discrete perturbation would help to complement the measurements. For now, these measurements serve to provide a unique data set with new information on the hypersonic receptivity process.

\section{Acknowledgments}

This research was funded by AFOSR Grant FA9550-12-1-0167 and the NASA Pathways Intern Employment Program. Special thanks are given here to Professor Steven H. Collicott for designing the laser perturbation-generating optical system and to Dr. Bradley M. Wheaton and Dr. Thomas J. Juliano for the initial design of the flared cone model. Professor Rolf Radespiel is acknowledged here for suggesting the use of an offset perturbation for these studies.

\section{References}

\footnotetext{
${ }^{1}$ Smith, A. and Gamberoni, N., "Transition, Pressure Gradient and Stability Theory," Tech. rep., Douglas Aircraft Division, Aug 1956, Report No. ES 26388.

${ }^{2}$ van Ingen, J. L., "The $e^{N}$ method for transition prediction. Historical review of work at TU Delft," AIAA Paper 20083830, Jun 2008.

${ }^{3}$ Mack, L. M., "Linear stability theory and the problem of supersonic boundary-layer transition," AIAA Journal, Vol. 13, No. 3, Mar 1975, pp. 278-289.

${ }^{4}$ Kendall, J. M., "Wind Tunnel Experiments Relating to Supersonic and Hypersonic Boundary-Layer Transition," AIAA Journal, Vol. 13, No. 3, Mar 1975, pp. 290-299.

${ }^{5}$ Morkovin, M. V., "Note on the Assessment of Flow Disturbances at a Blunt Body Traveling at Supersonic Speeds Owing to Flow Disturbances in Free Stream," Journal of Applied Mechanics, Vol. 27, No. 2, Jun 1960, pp. 223-229.

${ }^{6}$ Balakumar, P., "Receptivity of a Supersonic Boundary Layer to Acoustic Disturbances," AIAA Journal, Vol. 47, No. 5, May 2009, pp. 1069-1078.

${ }^{7}$ Balakumar, P., "Receptivity of Hypersonic Boundary Layers to Distributed Roughness and Acoustic Waves," AIAA Paper 2013-0082, Jan 2013.

${ }^{8}$ Balakumar, P. and Kegerise, M. A., "Receptivity of Hypersonic Boundary Layers to Acoustic and Vortical Disturbances," AIAA Paper 2011-0371, Jan 2011.

${ }^{9}$ Fedorov, A. V. and Kozlov, M. V., "Receptivity of high-speed boundary layer to solid particulates," AIAA Paper 20113925, Jun 2011.

${ }^{10}$ Mahesh, K., Lele, S. K., and Moin, P., "The influence of entropy fluctuations on the interaction of turbulence with a shock wave," Journal of Fluid Mechanics, Vol. 334, 1997, pp. 353-379.

${ }^{11}$ Fedorov, A. V., Ryzhov, A. A., and Soudakov, V. G., "Numerical and theoretical modeling of supersonic boundary-layer receptivity to temperature spottiness," AIAA Paper 2011-3077, Jun 2011.

${ }^{12}$ Huang, Y. and Zhong, X., "Numerical Study of Laser-Spot Effects on Boundary-Layer Receptivity for Blunt CompressionCones in Mach-6 Freestream," AIAA Paper 2010-4447, Jun 2010.

${ }^{13}$ Huang, Y. and Zhong, X., "Numerical Study of Freestream Hot-Spot Perturbation on Boundary-Layer Receptivity for Blunt Compression-Cones in Mach-6 Flow," AIAA Paper 2011-3078, Jun 2011.

${ }^{14}$ Huang, Y. and Zhong, X., "Parametric Study of Boundary-Layer Receptivity to Freestream Hot-Spot Perturbation over a Blunt Compression Cone," AIAA Paper 2014-774, Jan 2014.

${ }^{15}$ Maslov, A. A., Shiplyuk, A. N., Sidorenko, A. A., and Arnal, D., "Leading-edge receptivity of a hypersonic boundary layer on a flat plate," Journal of Fluid Mechanics, Vol. 426, 2001, pp. 73-94.

${ }^{16}$ Schmisseur, J. D., Collicott, S. H., and Schneider, S. P., "Laser-Generated Localized Freestream Perturbations in Supersonic and Hypersonic Flows," AIAA Journal, Vol. 38, No. 4, Apr 2000, pp. 666-671.

${ }^{17}$ Salyer, T. R., Laser Differential Interferometry for Supersonic Blunt Body Receptivity Experiments, Ph.D. thesis, School of Aeronautics \& Astronautics, Purdue University, West Lafayette, IN, May 2002.

${ }^{18}$ Heitmann, D., Radespiel, R., and Kähler, C., "Investigation of the response of a hypersonic boundary layer to controlled acoustic disturbances," AIAA Paper 2010-0536, Jan 2010.

${ }^{19}$ Chou, A., Schneider, S. P., and Kegerise, M. A., "Characterization of a Laser-Generated Perturbation in High-Speed Flow for Receptivity Studies," AIAA Paper 2014-0773, Jan 2014.
} 
${ }^{20}$ Schneider, S. P., "Flight Data for Boundary-Layer Transition at Hypersonic and Supersonic Speeds," Journal of Spacecraft and Rockets, Vol. 36, No. 1, Jan-Feb 1999, pp. 8-20.

${ }^{21}$ Schneider, S. P., "Effects of High-Speed Tunnel Noise on Laminar-Turbulent Transition," Journal of Spacecraft and Rockets, Vol. 38, No. 3, May-Jun 2001, pp. 323-333.

${ }^{22}$ Schneider, S. P., "Development of Hypersonic Quiet Tunnels," Journal of Spacecraft and Rockets, Vol. 45, No. 4, Jul-Aug 2008, pp. 641-664.

${ }^{23}$ Juliano, T. J., Nozzle Modifications for High-Reynolds-Number Quiet Flow in the Boeing/AFOSR Mach-6 Quiet Tunnel, Master's thesis, School of Aeronautics \& Astronautics, Purdue University, West Lafayette, IN, Dec 2006.

${ }^{24}$ Steen, L. E., Characterization and Development of Nozzles for a Hypersonic Quiet Wind Tunnel, Master's thesis, School of Aeronautics \& Astronautics, Purdue University, West Lafayette, IN, Dec 2010.

${ }^{25}$ Morgan, C. G., "Laser-induced breakdown phenomena," Scientific Progress, Vol. 65, No. 257, Spring 1978, pp. 31-50.

${ }^{26}$ Collicott, S. H., "Initial Mach 6 LT Spot Maker Design Summary," Tech. rep., Purdue University, Aug 2010, Informal design report.

${ }^{27}$ Salyer, T. R., Collicott, S. H., and Schneider, S. P., "Characterizing Laser-Generated Hot Spots for Receptivity Studies," AIAA Journal, Vol. 44, No. 12, Dec 2006, pp. 2871-2878.

${ }^{28}$ Berridge, D. C., Generating Low-Pressure Shock Waves for Calibrating High-Frequency Pressure Sensors (Unpublished Dissertation), Ph.D. thesis, School of Aeronautics \& Astronautics, Purdue University, West Lafayette, IN, May 2015.

${ }^{29}$ Chou, A., Mach-6 receptivity measurements of laser-generated perturbations on a flared cone, Ph.D. thesis, School of Aeronautics \& Astronautics, Purdue University, West Lafayette, IN, Aug 2014.

${ }^{30}$ Wheaton, B. M., Juliano, T. J., Berridge, D. C., Chou, A., Gilbert, P. L., Casper, K. M., Steen, L. E., Schneider, S. P., and Johnson, H. B., "Instability and Transition Measurements in the Mach-6 Quiet Tunnel," AIAA Paper 2009-3559, Jun 2009.

${ }^{31}$ Sivasubramanian, J. and Fasel, H. F., "Nonlinear Stages of Transition and Breakdown in a Boundary Layer on a Sharp Cone at Mach 6," AIAA Paper 2012-0087, Jan 2012.

${ }^{32}$ Berridge, D. C., Chou, A., Ward, C. A., Steen, L. E., Gilbert, P. L., Juliano, T. J., Schneider, S. P., and Gronvall, J. E., "Hypersonic Boundary-Layer Transition Experiments in a Mach-6 Quiet Tunnel," AIAA Paper 2010-1061, Jan 2010.

${ }^{33}$ Chou, A., Ward, C. A., Letterman, L. E., Luersen, R. P., Borg, M. P., and Schneider, S. P., "Transition Research with Temperature-Sensitive Paint in the Boeing/AFOSR Mach-6 Quiet Tunnel," AIAA Paper 2011-3872, Jun 2011.

${ }^{34}$ Balakumar, P. and Kegerise, M. A., "Receptivity of Hypersonic Boundary Layers over Straight and Flared Cones," AIAA Paper 2010-1065, Jan 2010. 